\title{
Excess reactive oxygen species production mediates monoclonal antibody-induced human embryonic stem cell death via oncosis
}

\author{
Ji Yun Zheng ${ }^{1}$, Heng Liang Tan ${ }^{2}$, Paul Thomas Matsudaira ${ }^{1,3}$ and Andre Choo $0^{\star, 2,4}$
}

\begin{abstract}
Antibody-mediated cell killing has significantly facilitated the elimination of undesired cells in therapeutic applications. Besides the well-known Fc-dependent mechanisms, pathways of antibody-induced apoptosis were also extensively studied. However, with fewer studies reporting the ability of antibodies to evoke an alternative form of programmed cell death, oncosis, the molecular mechanism of antibody-mediated oncosis remains underinvestigated. In this study, a monoclonal antibody (mAb), TAG-A1 (A1), was generated to selectively kill residual undifferentiated human embryonic stem cells (hESC) so as to prevent teratoma formation upon transplantation of $\mathrm{hESC}$-derived products. We revealed that A1 induces $\mathrm{hESC}$ death via oncosis. Aided with high-resolution scanning electron microscopy (SEM), we uncovered nanoscale morphological changes in A1-induced hESC oncosis, as well as A1 distribution on $\mathrm{hESC}$ surface. A1 induces $\mathrm{hESC}$ oncosis via binding-initiated signaling cascade, most likely by ligating receptors on surface microvilli. The ability to evoke excess reactive oxygen species (ROS) production via the Nox2 isoform of nicotinamide adenine dinucleotide phosphate (NADPH) oxidase is critical in the cell death pathway. Excess ROS production occurs downstream of microvilli degradation and homotypic adhesion, but upstream of actin reorganization, plasma membrane damage and mitochondrial membrane permeabilization. To our knowledge, this is the first mechanistic model of mAb-induced oncosis on hESC revealing a previously unrecognized role for NAPDH oxidase-derived ROS in mediating oncotic hESC death. These findings in the cell death pathway may potentially be exploited to improve the efficiency of $A 1$ in eliminating undifferentiated $h E S C$ and to provide insights into the study of other mAb-induced cell death.
\end{abstract}

Cell Death and Differentiation (2017) 24, 546-558; doi:10.1038/cdd.2016.164; published online 20 January 2017

Monoclonal antibodies (mAbs) have been widely used to eliminate undesired cells via various mechanisms, including antibody-dependent cell-mediated cytotoxicity (ADCC), complement-dependent cytotoxicity (CDC) and programmed cell death (PCD). Unlike the Fc-dependent mechanism of ADCC and CDC, certain antibody-antigen interaction can evoke direct PCD via apoptosis or oncosis. Antibodies can induce apoptosis via three major pathways, namely, antagonizing ligand-receptor signaling, ${ }^{1-3}$ crosslinking antigen ${ }^{4,5}$ and binding to surface receptors that transduce proapoptotic signals. ${ }^{6-8}$ Unlike apoptosis that has been extensively studied, the mechanism of oncosis remains unclear. Nevertheless, features of oncosis include rapid cell death, plasma membrane damage and cell swelling. ${ }^{9-11}$

Previously, our group reported the specific killing of undifferentiated human embryonic stem cells (hESC) by mAb84 via oncosis, thus preventing teratoma formation in hESC-based therapy. ${ }^{12,13}$ The authors postulated that the perturbation of actin-associated proteins facilitated the formation of plasma membrane pores via pentameric (IgM) mAb84mediated oligomerization of surface antigens. ${ }^{13}$ However, its mechanism of action remained unclear. More recently, our group generated another mAb, TAG-A1 (A1), which also kills
hESC via oncosis. However, as A1 is an IgG, it is unlikely to oligomerize antigens despite forming membrane pores. Hence, the central challenge is to identify the mechanism in the cell death pathway that elicit these features and potentially use it to augment the cytotoxic effect of mAbs.

In this study, we demonstrated that A1 specifically kills hESC via oncosis. Importantly, excess reactive oxygen species (ROS) production was deemed critical in A1 binding-initiated death signaling pathway. ROS was generated from nicotinamide adenine dinucleotide phosphate (NADPH) oxidase, and independent of mitochondrial impairment. It occurs downstream of microvilli degradation and homotypic adhesion, upstream of actin reorganization and plasma membrane damage. Based on the findings, we proposed a mechanistic model for A1-induced hESC oncosis.

\section{Results}

In vitro characterization of A1 on human pluripotent stem cells. From a panel of mAbs generated against hESC, A1 was shortlisted based on its ability to bind (Figure 1a) and kill (Figure 1b) undifferentiated hESC and hiPSC. The specificity

${ }^{1}$ Mechanobiology Institute (MBI), National University of Singapore (NUS), T-Lab, No. 10-01, 5A Engineering Drive 1, Singapore 117411, Singapore; ${ }^{2}$ Stem Cell 1 Group, Bioprocessing Technology Institute (BTI), Agency for Science, Technology and Research (A*STAR), 20 Biopolis Way, No. 06-01 Centros, Singapore 138668, Singapore; ${ }^{3}$ Department of Biological Science, Faculty of Science, National University of Singapore (NUS), 14 Science Drive 4, Singapore 117543, Singapore and ${ }^{4}$ Department of Biomedical Engineering, Faculty of Engineering, National University of Singapore (NUS), 9 Engineering Drive 1, Singapore 117575, Singapore

${ }^{*}$ Corresponding author: A Choo, Stem Cell 1 Group, Bioprocessing Technology Institute (BTI), Agency for Science, Technology and Research (A*STAR), 20 Biopolis Way, No. 06-01 Centros, Singapore 138668, Singapore. Tel: +65 64788856; Fax: +65 64789561; E-mail: andre_choo@ bti.a-star.edu.sg

Received 01.8.16; revised 03.11.16; accepted 25.11.16; Edited by RA Knight; published online 20.1.2017 
a

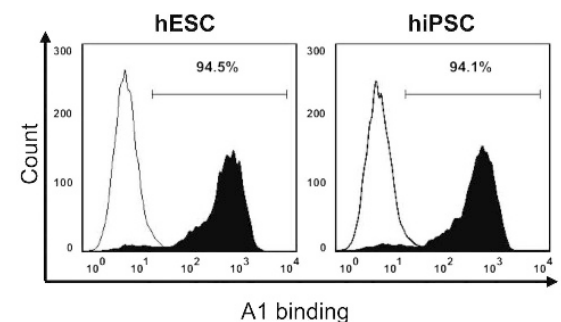

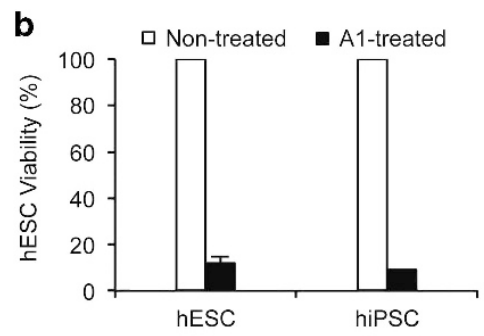

C

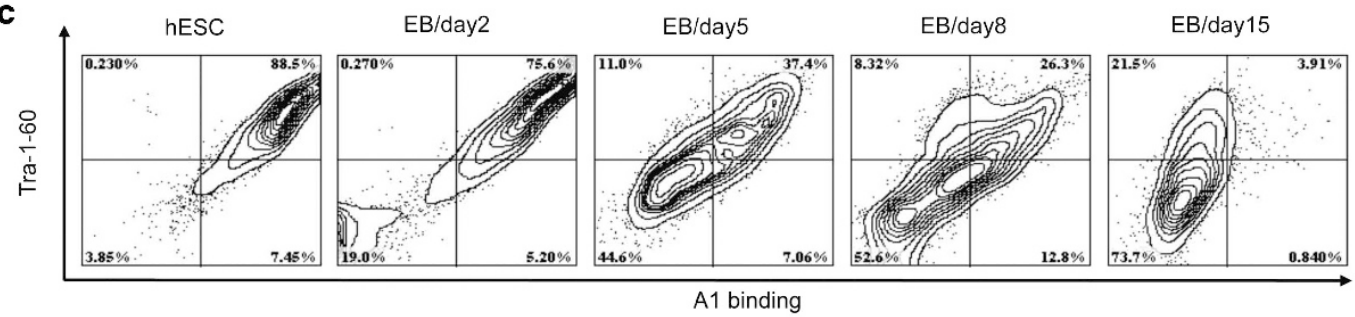

d
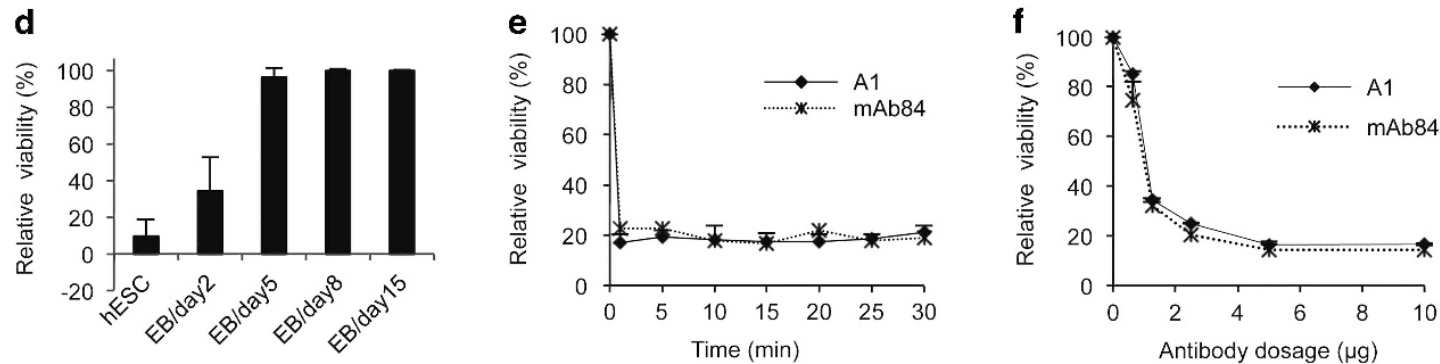

g

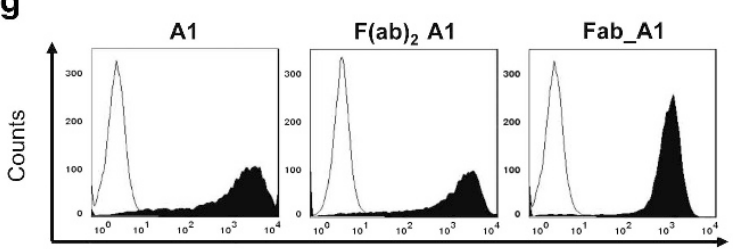

Binding to hESC (anti-kappa light chain specific)

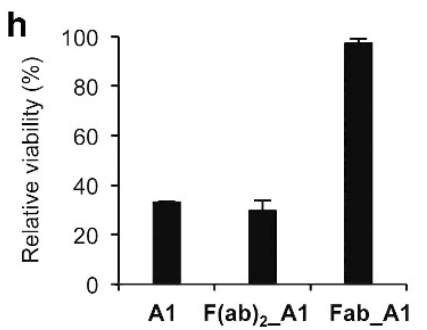

Figure 1 In vitro characterization of A1 on hESC. (a) A1 binds to and (b) kills both hESC (HES-3) and hiPSC (ESIMR90). A total of $2 \times 10^{5}$ cells (100 $\mu$ l) were incubated with $5 \mu \mathrm{g}$ of $\mathrm{A} 1$ for $45 \mathrm{~min}$ at $4{ }^{\circ} \mathrm{C}$. (c) Loss of $\mathrm{A} 1$ binding is correlated with loss of pluripotency marker (Tra-1-60) expression on spontaneously differentiated EBs. Cells were dual stained with A1 and anti-Tra-1-60 antibody. (d) A1 killing on undifferentiated hESC and spontaneously differentiated EBs at different stages. (e) A1 kills hESC within 1 min of incubation. A total of $2 \times 10^{5} \mathrm{cells}(100 \mu \mathrm{l})$ were treated with $5 \mu \mathrm{g}$ of $\mathrm{A} 1$ over different time periods at $4{ }^{\circ} \mathrm{C}$. (f) $\mathrm{A} 1 \mathrm{kills} \mathrm{hESC}$ in a dosage-dependent manner. A total of $2 \times 10^{5} \mathrm{cells}$ $(100 \mu l)$ were incubated with different amounts of $\mathrm{A} 1$ for 45 min at $4{ }^{\circ} \mathrm{C}$. (g) Binding and (h) killing on hESC were compared between $\mathrm{A} 1$, F(ab) ${ }_{2} \mathrm{~A} 1$ and Fab_A1. Antibody binding to hESC was detected with FITC-conjugated $\kappa$ light-chain-specific antibody. Open histogram represents no treatment control and shaded histogram represents antibody-treated cells. Cell viability was assessed via PI uptake by flow cytometry analysis, unless otherwise stated. Data are represented as mean \pm S.E.M.

of A1 was assessed on hESC-derived embryoid bodies (EBs) at different stages of spontaneous differentiation. A1 binding to cells was downregulated along with the loss of pluripotency marker (Tra-1-60) expression (Figure 1c). Concomitantly, a complete loss of A1 killing on differentiating cells was observed after 5 days (Figure 1d). Hence, the selective cytotoxicity of A1 on human pluripotent stem cell (hPSC) is beneficial for the removal of residual undifferentiated hPSC from differentiated cell products before transplantation.

A1 kills undifferentiated hESC within $1 \mathrm{~min}$ of incubation (Figure 1e) and in a dosage-dependent manner (Figure 1f), comparable to previously reported mAb84. ${ }^{12}$ Interestingly, both Fab_A1 and F(ab) 2_A1 bind to hESC (Figure 1g) but only
$\mathrm{F}(\mathrm{ab})_{2}$ A $\mathrm{A}$ retained $\mathrm{hESC}$ killing (Figure $1 \mathrm{~h}$ ). Hence, bivalency, but not Fc-domain, is essential for A1 killing on hESC.

A1 recognizes an 0 -linked glycan epitope containing glycan motif (Fuca1-2Gal $\beta$ 1-3GIcNAc $\beta$ 1-3Gal $\beta$ 1) on hESC antigens. After immunoprecipitation (IP) and immunoblotting with $\mathrm{A} 1$, an antigen smear from 35 to $200 \mathrm{kDa}$ was revealed (Figure $2 \mathrm{a}$ ), suggesting that $\mathrm{A} 1$ may be recognizing glycans on multiple antigens. This was confirmed by using sodium periodate to open sugar rings $^{14}$ on immunoprecipitated antigens. Compared with the non-treated control, A1 binding to the entire smear was abolished after periodate treatment (Figure 2a). 
a

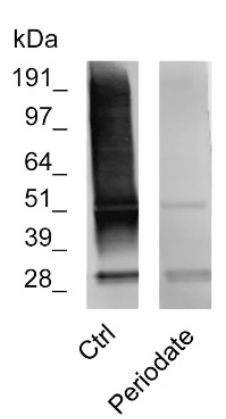

b

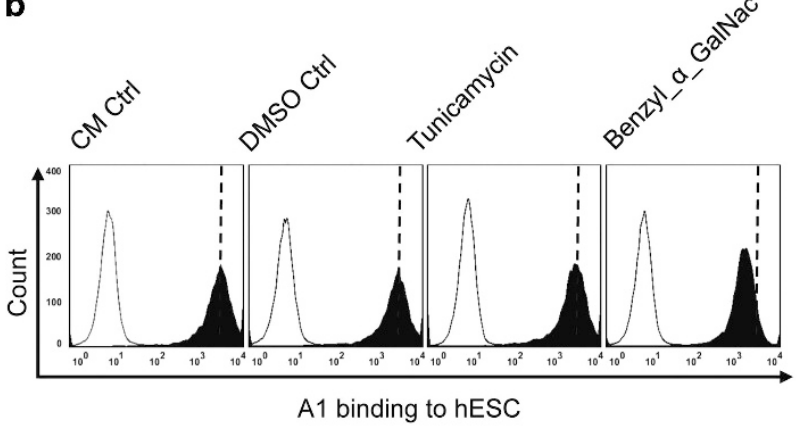

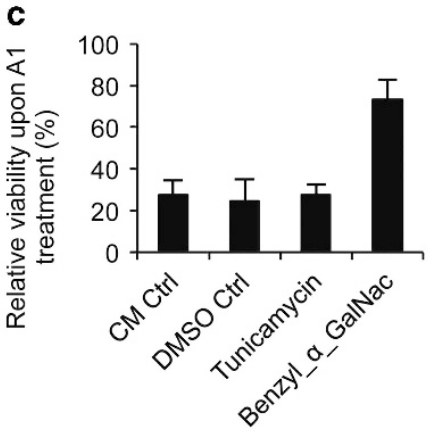
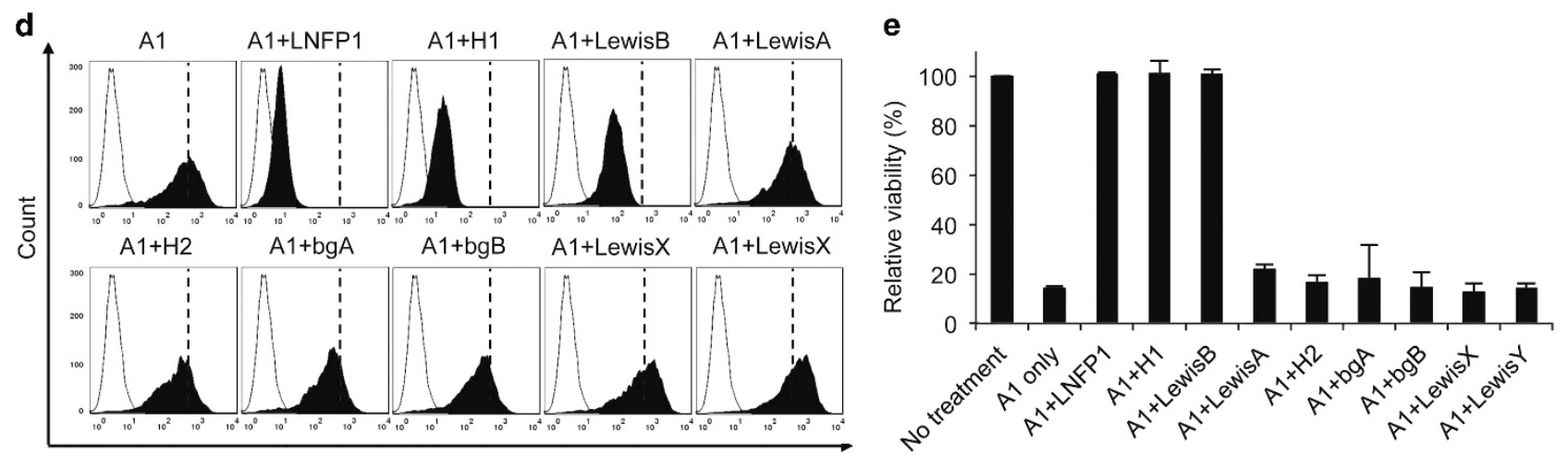

A1 binding to $h E S C$

Figure 2 Antigen targets of A1 on hESC surface. (a) A1 binds to glycans on multiple target antigens on hESC. A1 binding (b) and killing (c) on hESC were partially blocked upon B-GalNac treatment $(4 \mathrm{mM}, 24 \mathrm{~h})$, but not tunicamycin treatment $(5 \mu \mathrm{g} / \mathrm{ml}, 24 \mathrm{~h})$. A1 binding (d) and killing (e) on hESC were blocked by LNFP1, H1 and LewisB. Five micrograms of A1 was preincubated with the nine different sugars $(2 \mathrm{mM})$ before treatment with $2 \times 10^{5}$ cells $(100 \mu l)$ for 45 min at $4{ }^{\circ} \mathrm{C}$. Open histogram represents no treatment control and shaded histogram represents antibody-treated cells. Data are represented as mean \pm S.E.M. See also Supplementary Figures S1 and S2

Subsequently, tunicamycin ${ }^{15}$ and benzyl-a-GalNac $(\mathrm{B}-\mathrm{GalNac})^{16}$ were used to inhibit $\mathrm{N}$-glycosylation and O-glycosylation, respectively, in hESC culture, which was confirmed by the lost of concanavalin $A$ and anti-Tra-1-60 binding to inhibitor-treated hESC (Supplementary Figures $S 1 A$ and $B$ ). When $A 1$ was tested on both cell populations, $A 1$ binding to hESC was only downregulated in B-GalNac-treated cells (Figure 2b). Pluripotency of inhibitor-treated hESC was not affected by B-GalNac as determined by the RNA expression levels of various markers (Supplementary Figure S2B). Concomitantly, cell viability after A1 treatment was significantly higher with B-GalNac treatment compared with the control groups (Figure $2 \mathrm{c}$ ), indicating that binding to O-linked glycans is essential for A1 killing on hESC.

To determine the glycan epitope that $A 1$ recognizes on hESC, A1 binding and killing on hESC was examined after preincubation with nine sugars of the non-sialylated blood group antigens. A significant decrease in A1 binding to hESC was observed upon preincubation with type-1 $\mathrm{H}(\mathrm{H} 1)$, lacto- $N$ fucopentaose I (LNFP1) and LewisB (Figure 2d), indicating competitive binding for the epitope on hESC. Among the nine sugars, H1, LNFP1 and LewisB share a common glycan motif Fuca1-2Gal $\beta 1-3 G \mid c N A c \beta 1-3 G a l \beta 1$ (Supplementary Figure S1C). Moreover, upon preincubation with any of these three sugars, A1 killing on hESC was abolished (Figure 2e). Taken together, A1 binds to an O-linked glycan epitope containing Fuca1-2Gal $\beta 1-3 \mathrm{GlcNAc} \beta 1-3 \mathrm{Gal} \beta 1$ to elicit cytotoxicity on hESC.

A1 induces hESC death via oncosis. To further investigate the lethal effect of $A 1$ on hESC, changes to hESC morphology was examined under scanning electron microscopy (SEM). In general, non-treated hESC had uniform shape, rich microvilli and intact plasma membrane (Figure 3a). Upon A1 treatment, a five-staged change in hESC morphology was summarized (Figures $3 b-f)$. However, because of the rapid killing of hESC by $A 1$, more than one stage was observed simultaneously. At stage 1, A1-treated hESC typically displayed partially degraded or shortened microvilli with enlarged cell volume, whereas the membrane integrity remained intact (Figure $3 b$ ). Some fused microvilli suggested the transition from stage 1 to stage 2. At stage 2, varied size membrane pores were formed (Figure 3c). Another feature was the appearance of one or more circular-shaped areas of $\sim 2 \mu \mathrm{m}$ that were surrounded by fused microvilli. Within this area, microvilli were absent with the presence of cytoskeleton-like structures. At stage 3, microvilli further shortened or even completely disappeared (Figure 3d). Unlike stage 2, in the circular area, both the surrounding fused microvilli and the centered cytoskeletonlike structures disappeared. Moreover, larger membrane pores were formed. At stage 4, membrane damage was more pronounced and likely originated from the damaged 
a

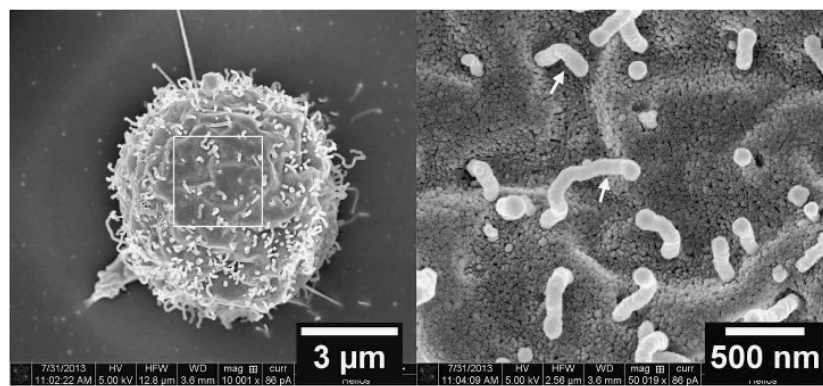

C A1-treated hESC, stage 2

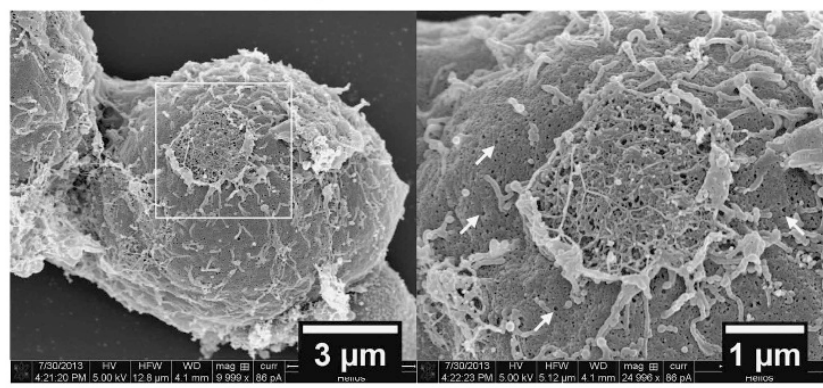

e A1-treated hESC, stage 4

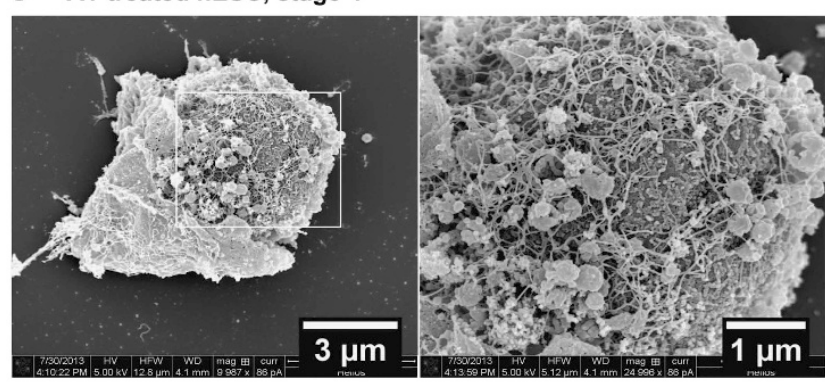

\section{b A1-treated hESC, stage 1}

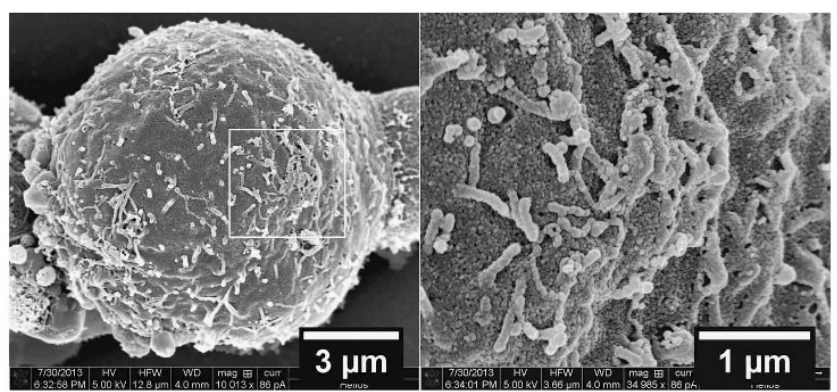

d A1-treated hESC, stage 3

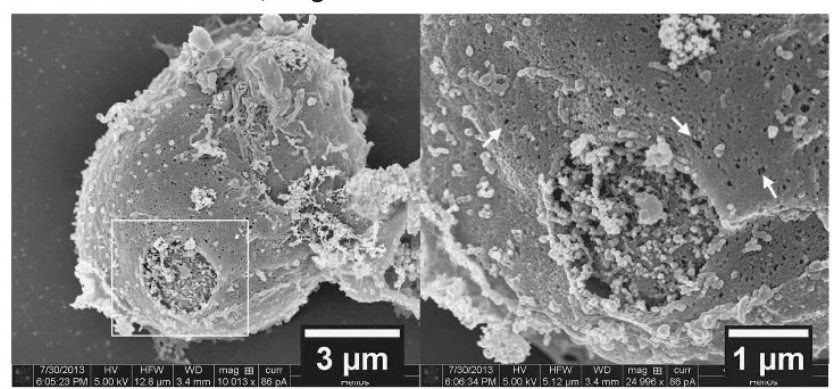

f A1-treated hESC, stage 5

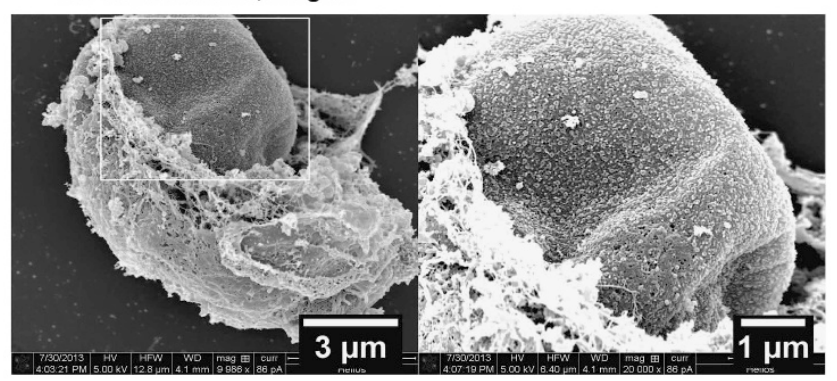

g

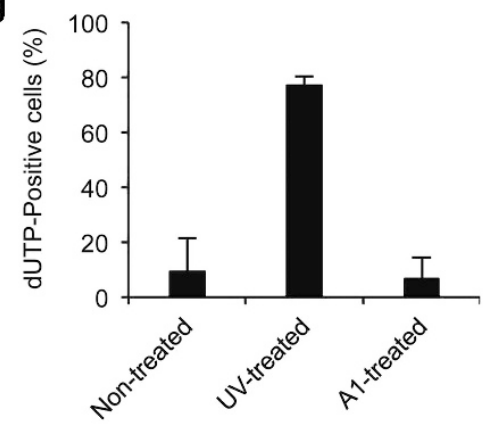

h

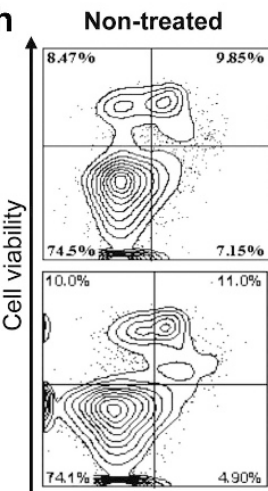

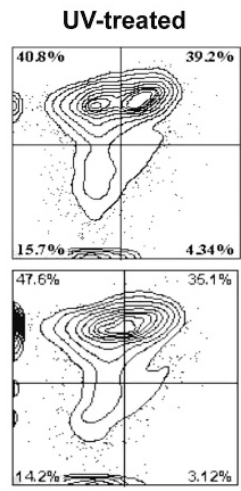

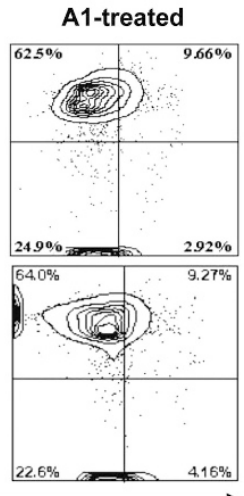

Caspase $3 \& 7$

Caspase 9

Caspase activity

Figure 3 A1 induces hESC death via oncosis. (a-f) Observation of hESC structure and cell surface morphology under S.E.M. Left: Overview of a single hESC; right: highlighted area in higher magnification. (a) hESC without A1 treatment. Arrows indicates microvilli. (b-f) Five-staged morphological changes of A1-treated hESC within 30 min. Arrows indicate pore formation. Fused microvilli at stage 1, circular area at stages 2 and 3, cytoskeleton-covered nucleus at stage 4 and exposed nucleus at stage 5 are highlighted in boxes. (g) The extent of DNA fragmentation measured by TUNEL assay. Increase in dUTP fluorescence intensity was correlated to increased DNA fragmentation. (h) Scatter plot of caspases activity and cell death. The activity of caspases (caspases 3, 7 and 9) was compared between non-treated hESC, UV-treated hESC and A1-treated $\mathrm{hESC}$. Fluorescence-labeled inhibitor of caspase reagents were used to specifically detect activated caspase. To correlate cell death with caspase activity, cells were costained with a cell-impermeable dye, 7-AAD (7-aminoactinomycin D), to identify dead cells. Fluorescence intensity of caspase reagents and 7-AAD were analyzed by flow cytometry. Lower left (LL) quadrant corresponds to viable cells without activated caspase activity, right quadrants (upper and lower) to activated caspase activity and upper quadrants (right and left) to dead cells. Data are represented as mean \pm S.E.M. 
circular area of stage 3 revealing the cytoskeleton structure and nucleus (Figure $3 e$ ). Last, at stage 5 , severely damaged membrane peeled off exposing the nucleus. The progressive morphological changes suggested that A1-induced hESC death is likely associated with altered stability of microvilli and cytoskeleton structures.

Observed features of A1-induced hESC death, including rapid cell death, cell swelling and plasma membrane damage, suggest that it is unlikely a consequence of apoptosis, which is a slow process (12-24h) featured with cellular shrinkage, condensation of nucleus, formation of apoptotic bodies, increased caspase activity and DNA fragmentation. ${ }^{17}$ On the contrary, these features resembled the hallmarks of oncosis. ${ }^{11,13}$ This observation was reiterated by TUNEL (terminal deoxynucleotidyl transferase-mediated dUTP nickend labeling) and caspase assays. A1-treated hESC did not exhibit significant increase in DNA fragmentation (Figure $3 \mathrm{~g}$ ). Caspase activity (caspases 3, 7 and 9) measured for both A1treated and non-treated hESC were also comparable and at minimal basal levels (right quadrants) while there was increased activated caspases for ultraviolet (UV)-treated hESC, especially in the upper right quadrant, which corresponds to apoptotic cells (Figure 3h). In contrast, for A1-treated hESC, there was an increase in the dead cell population within the upper left quadrant compared with the non-treated hESC. Taken together, we concluded that $A 1$ kills hESC via oncosis.

A1 binds uniformly on hESC and elicits homotypic adhesion and actin reorganization. To better understand how $A 1$ binding to hESC leads to cell death, we examined the distribution pattern of A1 on hESC surface under SEM aided with gold-conjugated secondary antibodies. No nonspecific binding of secondary antibodies was observed in non-treated control (Figure 4a). In A1-treated hESC, A1 particles were uniformly distributed across the cell surface (Figure $4 \mathrm{~b}$ ). Previously, in mAb84-induced hESC death, gold-labeled antibody aggregates were interpreted as antibodies clustering the surface antigens to form pores throughout the plasma membrane. ${ }^{13}$ However, in our study, there was no colocalization observed between the $\mathrm{A} 1$ binding pattern and distribution of membrane pores. Instead, clusters of gold particle were more likely a result of microvilli fusion (Figure 4c). These observations suggest that formation of membrane pores was not a result of external mechanical force from $A 1$ binding, but rather a binding-initiated signaling cascade. The minimally required bivalency of $A 1$ (Figure $1 \mathrm{~h}$ ) might contribute to the signaling pathway by ligating receptors on hESC. Surprisingly, we also observed that the majority of $A 1$ binding was on surface microvilli (Figure $4 d$ ), suggesting that A1-induced death signaling might be initiated through receptors on microvilli.

Upon A1 binding, formation of hESC aggregates/homotypic adhesion was observed (Figure 5a), which was also reported in other mAb-induced oncosis. ${ }^{18,19}$ Intriguingly, the difference in the ability of $F(a b)_{2} A 1$ and Fab_A1 to induce homotypic adhesion corresponded with the difference in their cytotoxicity on hESC (Figure 1h), indicating homotypic adhesion and cell death is functionally related.
Homotypic adhesion, microvilli degradation and plasma membrane damage all implied the involvement of actin cytoskeleton in A1-induced hESC death. Similar to mAb84induced hESC oncosis, ${ }^{13}$ significant reduction of four actinassociated proteins ( $a$-actinin, paxillin, talin and vinculin) in A1-treated hESC was observed (Figure 5b). Next, the effect of actin polymerization inhibitors (cytochalasin $B$, cytochalasin $D$ and latrunculin A) on A1-induced hESC death was investigated. Upon A1 treatment, inhibitor-treated hESC had a reduction in cell death compared with the non-treated controls (Figure 5c), suggesting a functional link between actin polymerization and $\mathrm{A} 1$-induced $\mathrm{hESC}$ death. Moreover, immunostaining revealed that $A 1$ treatment evoked the enrichment of F-actin at cell-cell adhesion sites and the relocalization of G-actin from the nucleus to the cytoplasm (Figure 5d). Taken together, these results demonstrated a close association between actin reorganization and $\mathrm{A} 1$ induced hESC death.

A1-induced hESC death is mediated by excess ROS production from NADPH oxidase. Previously, excess ROS production has been reported in several $\mathrm{mAb}$-induced cell death. $^{20-22}$ Studies involving an anti-CD20 mAb, GA101, targeting human B-lymphoma cells, demonstrated that depleting ROS with scavengers partially inhibited GA101induced cell death. ${ }^{23}$ Interestingly, GA101 also kills human B-lymphoma cells via a non-apoptotic pathway featuring homotypic adhesion and plasma membrane damage. ${ }^{18,24}$ Therefore, we hypothesized that ROS might also have an essential role in A1-induced hESC death.

Excess ROS production was detected via dihydroethidium (HE) staining upon A1 treatment (Figures 6a and b). This was also confirmed by carboxy-H2DCFDA staining (Supplementary Figure S3A). By dual-staining hESC with Sytox green (dead cells) and HE (ROS production), the direct correlation between excess ROS production and cell death was demonstrated (Figure 6c). Next, using ROS scavengers: Tiron (4,5-dihydroxy-1,3-benzenedisulfonic acid disodium salt monohydrate) and Tempol (4-hydroxy-2,2,6,6-tetramethylpiperidine 1-oxyl), which are well-characterized superoxide dismutase mimetics, ${ }^{25}$ the extent of cell killing by $A 1$ was compared. Pre-treatment of hESC with both Tiron and Tempol significantly inhibited A1-induced hESC death (Figure 6d), indicating that excess ROS production is required for $\mathrm{A} 1 \mathrm{killing}$ on hESC. The depletion of ROS by Tiron was found to correlate with the decrease in $\mathrm{A} 1$-induced cell death (Figure 6e), confirming the essential role of ROS in A1-induced hESC death. For both Tiron and Tempol, the mechanism of action is to convert superoxide $\left(\mathrm{O}^{2-}\right)$ to hydrogen peroxide, ${ }^{26}$ suggesting that superoxide is the form of ROS responsible for A1-induced hESC death.

Next, the upstream source of A1-induced superoxide production in hESC was explored. In living cells, the major sources of superoxide production are mitochondria and NADPH oxidases. $^{27}$ First, the activity of NADPH oxidase was estimated by measuring NADP/NADPH ratio. Upon activation of NADPH oxidase, NADPH is oxidized to NADP ${ }^{+}$ with the production of superoxide. A1-treated hESC has significantly higher NADP/NADPH ratio than non-treated hESC (Figure 7a). To confirm that NADPH oxidase is 
a

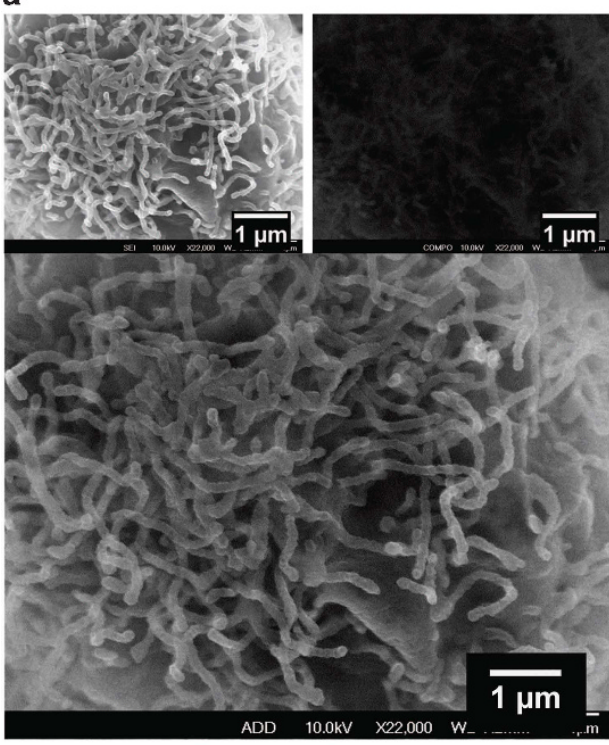

c

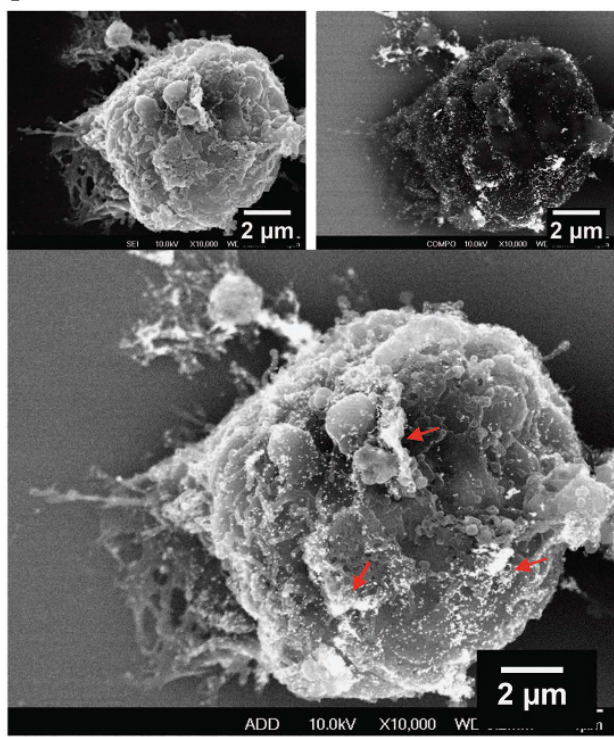

b

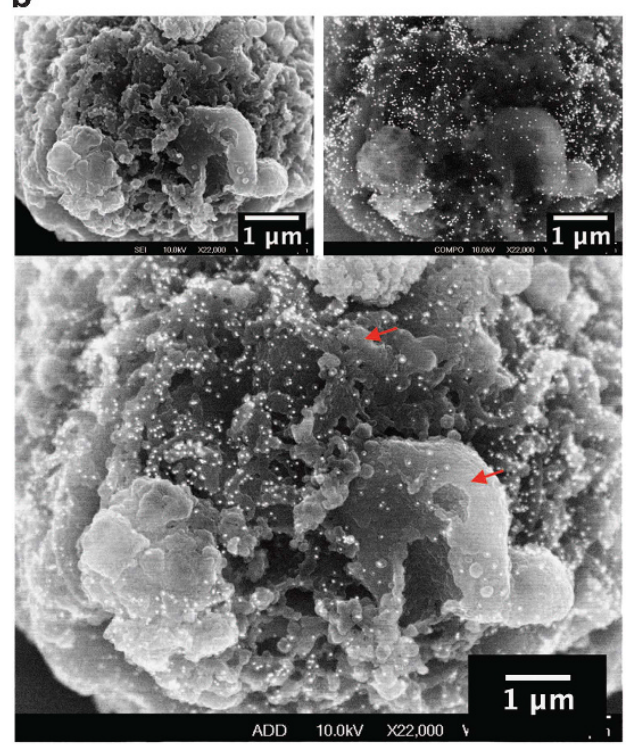

d
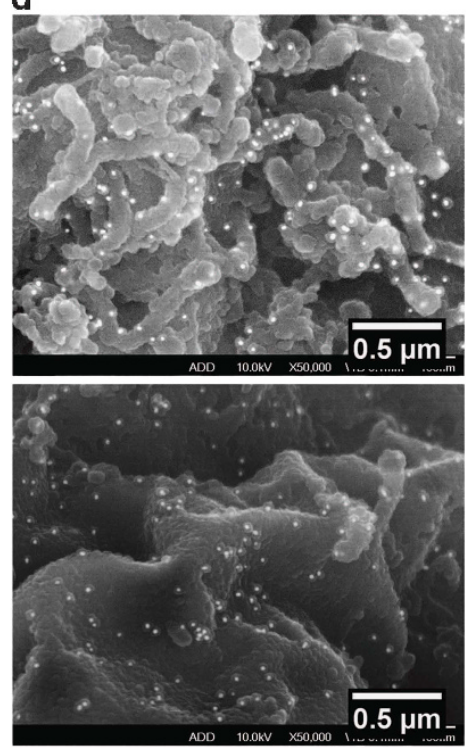

Figure 4 A1 distribution on $\mathrm{hESC}$ surface under immune-S.E.M. (a) No nonspecific binding of secondary antibodies on $\mathrm{hESC}$. (b and c) Observation of A1 binding and hESC morphology. Cell morphology was detected under secondary electron mode (upper left), whereas gold particle-labeled A1 were detected under backscattering mode (upper right). Overlay between cell morphology and A1 binding was viewed under combined mode (bottom). Arrows in (b) indicate membrane pores, which were not correlated with A1 binding pattern. Arrows in (c) indicate the correlation between A1 (gold particles) aggregates and fused microvilli or plasma membrane. (d) A1 mostly binds to surface microvilli, either elongated (upper panel) or shortened (lower panel)

responsible for superoxide production, three inhibitors of NADPH oxidase, diphenyleneiodonium chloride (DPI), mycophenolic acid (MPA) and apocynin (APO), were used. Pretreatment with these inhibitors significantly attenuated A1 killing on hESC (Figure 7b). Moreover, decrease in cell death directly corresponded to the inhibition of ROS production (Supplementary Figure S3B).

Among the seven isoforms of NADPH oxidase, only the activation of Nox1, Nox2 and Nox3 requires the assembly of cytosolic and membrane-bound subunits as well as Rac activation, which should be the only targets of MPA and APO. The role of Nox2 in $\mathrm{A} 1$-induced ROS production was first investigated. Two different small interfering RNAs (siRNAs) (siNox2_s3787 and siNox2_s3788) were used to downregulate Nox2 expression in hESC. Transfection efficiency was verified using qRT-PCR (Supplementary Figure S3C) and western blotting (Figure 7c). Expression of pluripotency marker Tra-1-60 on hESC after transfection was confirmed (Supplementary Figure S3D). With partial knockdown of Nox2, A1 binding was not affected (Supplementary Figure S3E), indicating that Nox2 is not an antigen target of A1. However, A1-induced hESC death was significantly attenuated (Figure 7d). Subsequently, the expression of Nox1 and Nox3 in hESC was assessed. Nox1 was not detected, whereas 
a

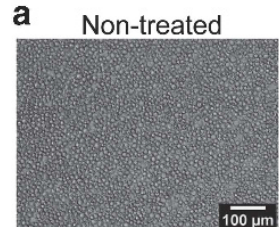

$\mathrm{F}(\mathrm{ab})_{2} \mathrm{~A} 1$

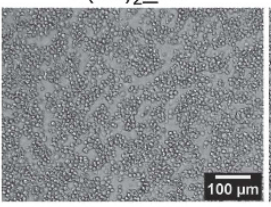

A1

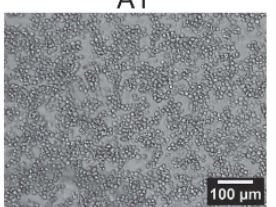

Fab_A1

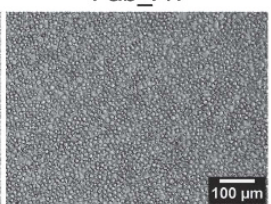

b

Vinculin

$\alpha-A c t i n i n$

Paxillin

GAPDH
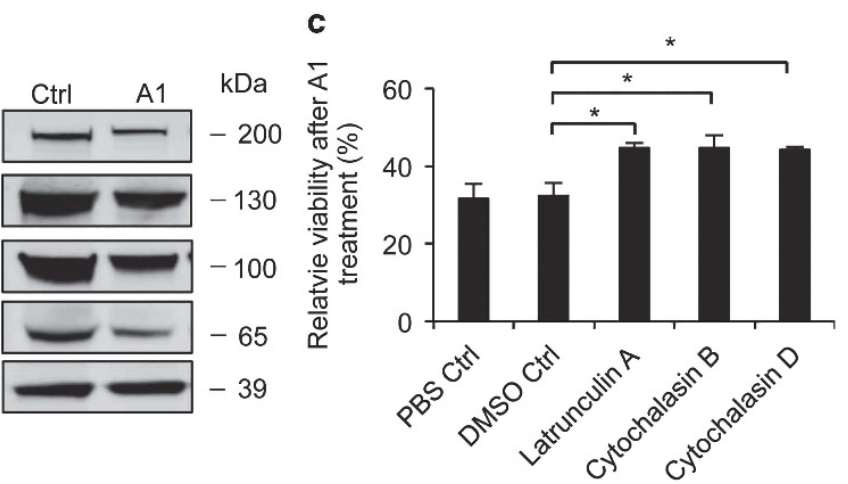

d
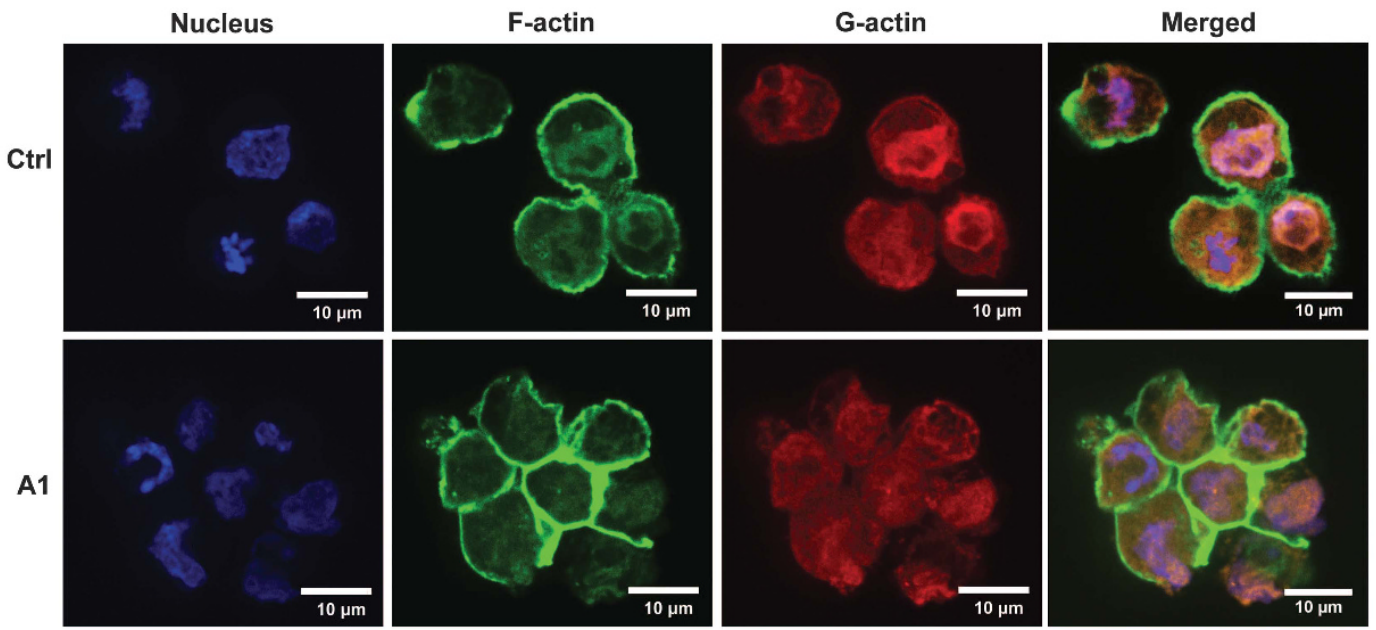

Figure 5 A1 binding on $\mathrm{hESC}$ elicits homotypic adhesion and actin reorganization. (a) Bivalent $\mathrm{A} 1$ and $\mathrm{F}(\mathrm{ab})_{2} \mathrm{~A} 1$ can induce $\mathrm{hESC}$ homotypic adhesion, but not monovalent Fab_A1. (b) Degradation of actin-associated proteins, namely, talin, vinculin, $\alpha$-actinin and paxillin, upon A1 treatment. Glyceraldehyde 3-phosphate dehydrogenase (GAPDH ) was used as an endogenous control. (c) A1 cytotoxicity on hESC was partially inhibited upon actin inhibitors treatment. $\mathrm{hESC}\left(2 \times 10^{5}\right.$ cells in $\left.100 \mu \mathrm{l}\right)$ were preincubated with the actin inhibitors: latrunculin $\mathrm{A}(4 \mu \mathrm{g})$, cytochalasin $\mathrm{B}(4 \mu \mathrm{g})$ and cytochalasin $\mathrm{D}(4 \mu \mathrm{g})$ for 5 min before $\mathrm{A} 1$ treatment. Data are represented as mean \pm S.E.M. ${ }^{*} P<0.05$, ${ }^{* *} P<0.01$ and ${ }^{* * *} P<0.001$. (d) $A 1$ treatment leads to enrichment of $F$-actin at cell-cell adhesion cites and $\mathrm{G}$-actin translocation from the nucleus to the cytoplasm. Representative images are shown. Nucleus was stained with Hoechst blue, F-actin was labeled with Alexa Fluor 488 phalloidin and G-actin was labeled with deoxyribonuclease I, Alexa Fluor 594 conjugate

Nox3 was detected around the predicted molecular weight of $51 \mathrm{kDa}$ (Supplementary Figure S4A) and distributed in both the cytoplasm and plasma membrane (Supplementary Figure S4B). Subsequently, Nox3 expression was transiently downregulated with siRNA. Transfection efficiency (Supplementary Figure S4C) and pluripotency of hESC after transfection (Supplementary Figure S4D) was confirmed. However, downregulation of Nox3 did not affect A1 killing on hESC (Figure 7e), suggesting that Nox3 is not a mediator of A1-induced hESC death.

As neither Nox2 knockdown nor inhibition of A1-induced hESC death was complete, it remained unclear whether Nox2 was the only mediator of A1-induced hESC death. Besides NADPH oxidase, excess superoxide production upon mitochondrial impairment has been reported in apoptosis. ${ }^{28}$ Upon A1 treatment, an increase in mitochondrial depolarization was detected by the potentiometric dye JC-1 (Supplementary Figure S4E), indicating that A1 treatment can induce hESC mitochondrial impairment. However, by downregulating Nox2 expression in hESC, JC-1 measured mitochondrial depolarization was reduced correlating to the attenuation in cell death
(Figure 7f), suggesting mitochondrial depolarization is a consequence of A1-induced Nox2 activation, and unlikely an alternative source of superoxide production. In summary, Nox2-NADPH oxidase is the source of A1-induced ROS production and it is essential for $A 1$ to elicit cytotoxicity on hESC.

ROS production occurs downstream of microvilli degradation and homotypic adhesion, upstream of actin reorganization. So far, several events have been observed during $\mathrm{A} 1$-induced $\mathrm{hESC}$ death including microvilli degradation, homotypic adhesion, actin reorganization, plasma membrane damage and ROS production. Given the functional significance of ROS production in A1-induced hESC death, we next investigated where along the pathway is ROS production effecting. Therefore, the effect of ROS depletion on these observed events was assessed. Tiron treatment did not inhibit homotypic adhesion induced by A1 (Figure 8a), suggesting ROS production occurs downstream of homotypic adhesion. Under SEM, Tiron-treated hESC also resulted in microvilli degradation upon A1 treatment (Figure 8b). 
a

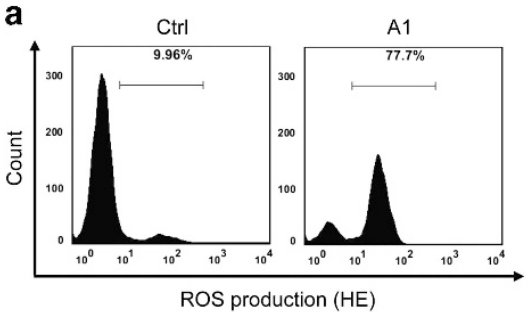

d

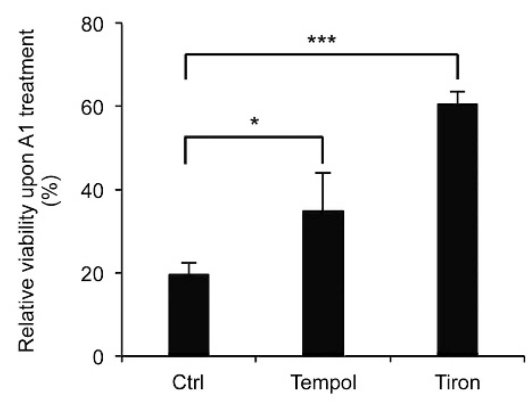

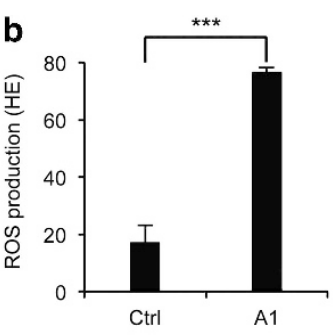

C

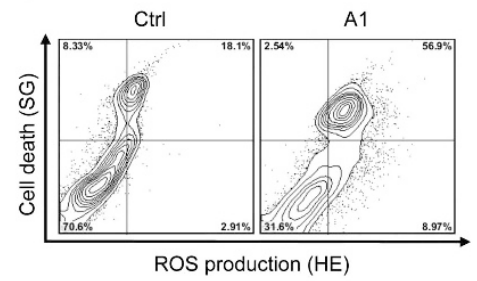

e

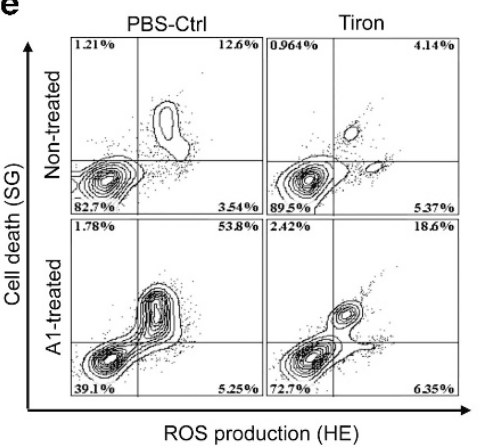

Figure 6 A1-induced hESC death is mediated by excess ROS production. (a) ROS production was measured via HE staining ( $25 \mu \mathrm{M})$, which emits red fluorescence when oxidized by ROS. (b) A1 treatment led to excess ROS production (measured by HE staining) in hESC. (c) A1-induced hESC death was directly correlated with excess ROS production. (d) ROS scavengers can partially block A1-induced hESC death. Before A1 treatment, cells were treated with phosphate-buffered saline (PBS) or with ROS scavengers (Tiron $50 \mathrm{mM}$ or Tempol $120 \mathrm{mM}$ ) for $1 \mathrm{~h}$. (e) Depletion of ROS production were correlated with the decrease in A1-induced hESC death. Data are represented as mean \pm S.E.M. ${ }^{*} P<0.05,{ }^{* *} P<0.01$ and ${ }^{* *} P<0.001$. See also Supplementary Figure S3
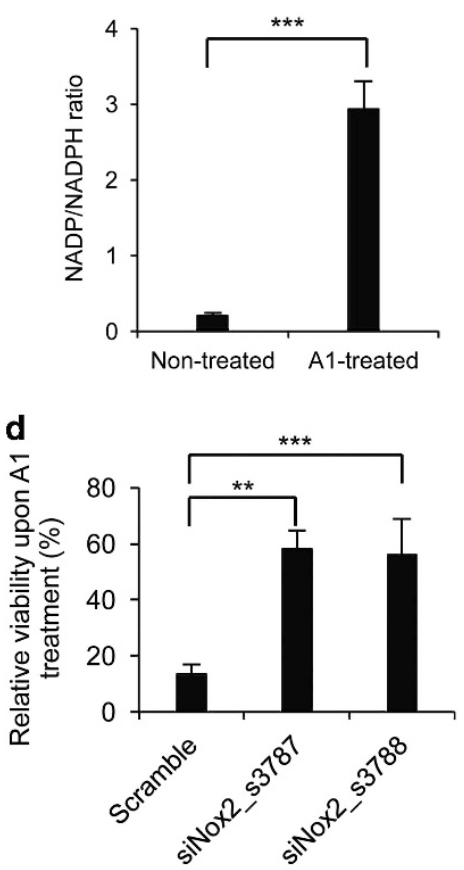

b

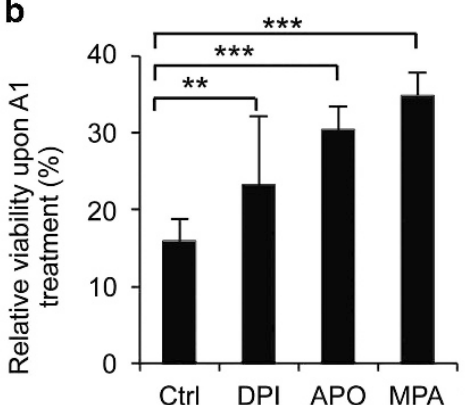

e

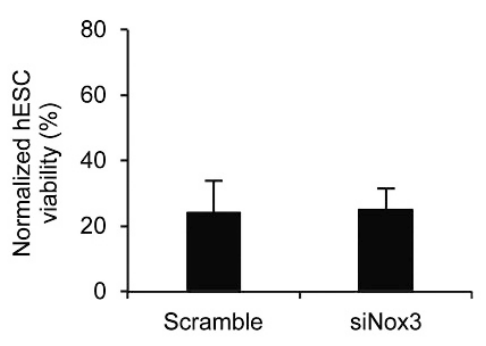

c
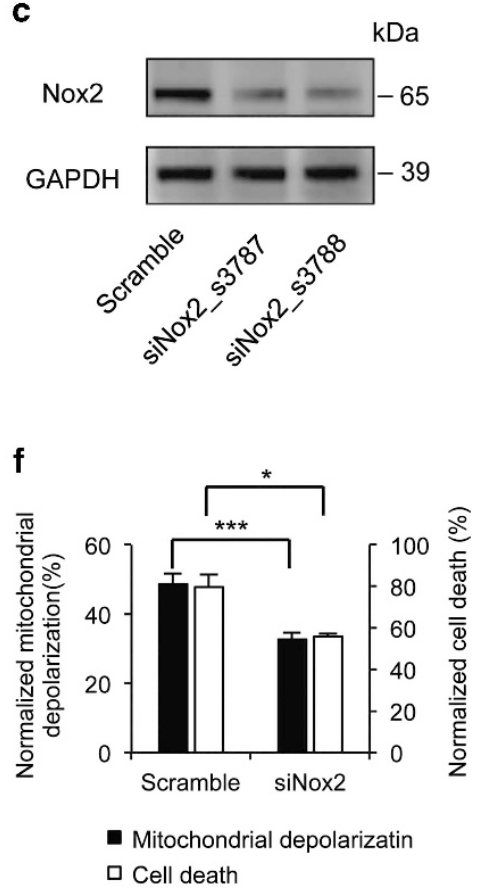

Figure 7 A1 induces excess ROS production from NADPH oxidase. (a) Activity of NADPH oxidase as measured via NADP ${ }^{+} / N_{A D P H}$ ratio in $\mathrm{hESC}$ significantly increase upon A1 treatment. (b) Inhibitors of NADPH oxidase can partially block A1-induced hESC death. Before A1 treatment, cells were treated with dimethyl sulfoxide (DMSO) or inhibitors of NADPH oxidase (DPI $240 \mathrm{mM}$, or APO $40 \mathrm{mM}$, or MPA $3.12 \mathrm{mM}$ ) for $1 \mathrm{~h}$. DPI abstracts an electron from NADPH oxidase which then inhibits electron transfer from NADPH to oxygen. ${ }^{51}$ MPA depletes cellular GTP and consequently inhibits Rac activation. ${ }^{52}$ APO prevents the translocation of cytosolic components to plasma membrane. ${ }^{53}$ (c) Knockdown of Nox2 was confirmed by western blotting. (d) Upon A1 treatment, Nox2-knockdown hESC showed higher cell viability. (e) Upon A1 treatment, cell viability of Nox3-knockdown hESC did not increase. (f) Mitochondrial membrane permeabilization as measured via potentiometric dye JC-1 is a consequence of A1-induced ROS production. Data are represented as mean \pm S.E.M. ${ }^{\star} P<0.05,{ }^{\star \star} P<0.01$ and ${ }^{* \star *} P<0.001$. See also Supplementary Figures S3 and S4 
However, Tiron-treated hESC retained a uniform cell size and morphology without plasma membrane damage (Figure 8b), indicating that ROS production lies downstream of microvilli degradation, and upstream of plasma membrane damage. In the presence of Tiron, actin inhibitors treatment did not significantly inhibit A1 killing (Figure 8c). More importantly, regardless of Tiron, treatment with actin inhibitors did not influence A1-induced ROS production (Figure 8d), indicating a

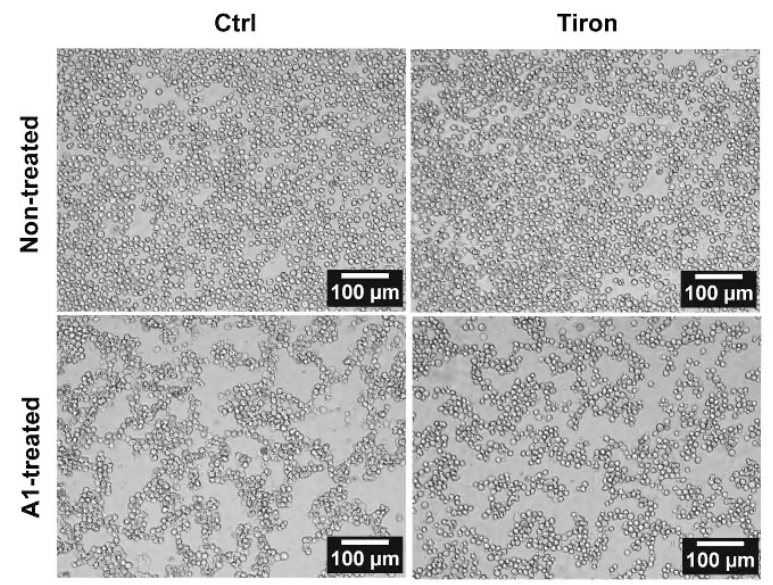

C

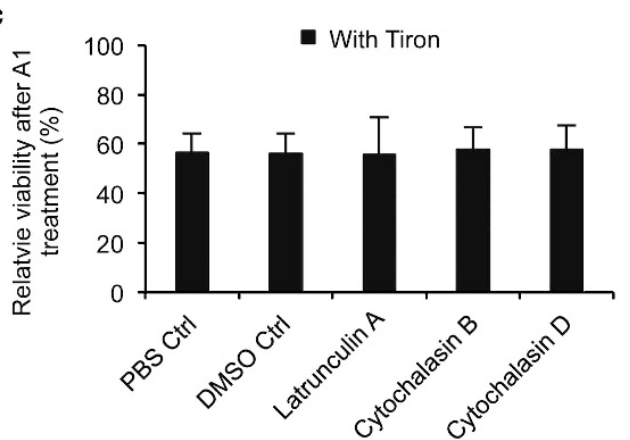

b
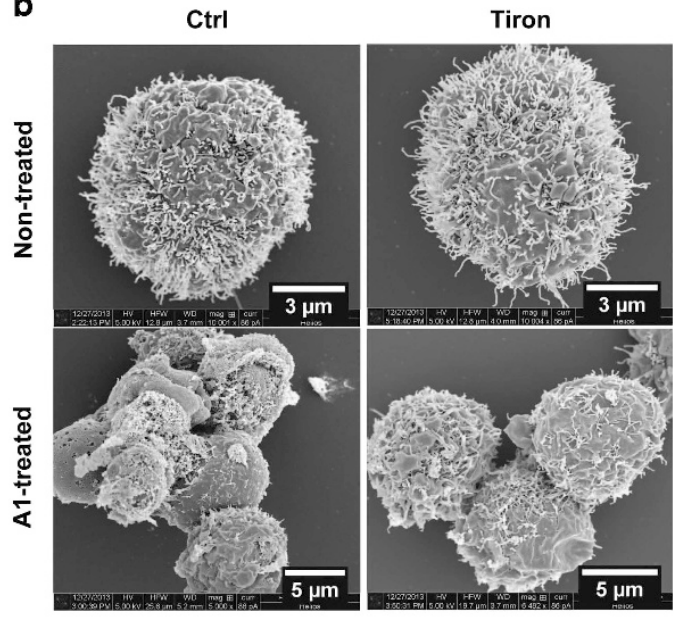

d

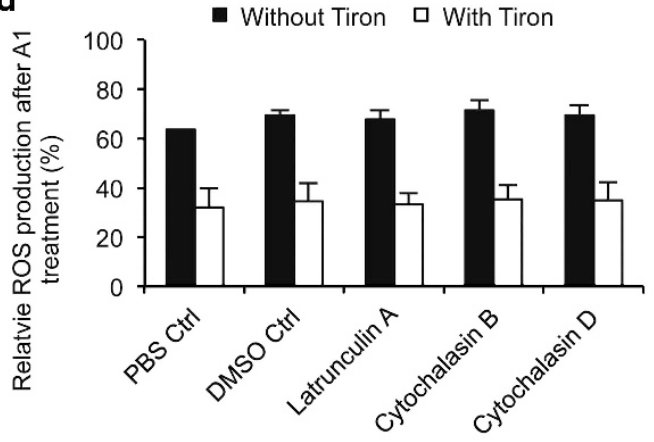

e

Bivalent A1 binds and ligates certain antigen receptors to initiate death signal

Microvilli degradation

Homotypic adhesion (HA)

$\downarrow$

Elevated ROS $\left(\mathrm{O}^{2-}\right)$ production by NADPH oxidase

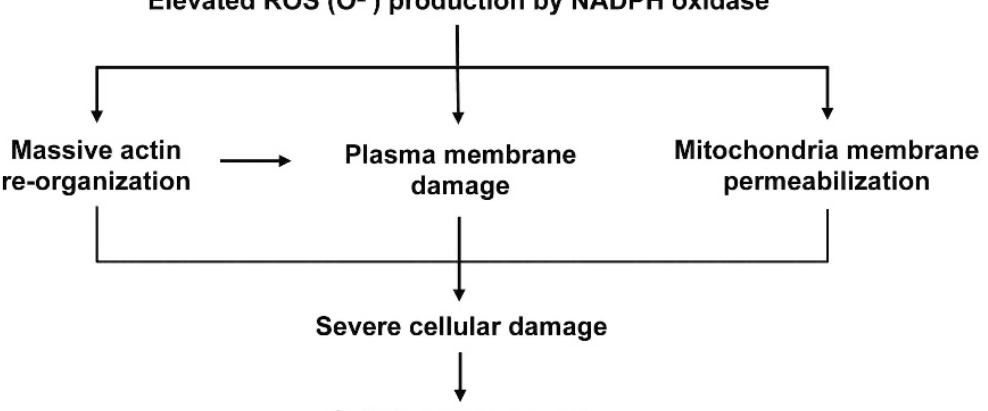

Cell death via oncosis

Figure 8 Delineated order of events in A1-induced hESC death. (a) ROS depletion by Tiron cannot prevent A1-induced homotypic adhesion. (b) ROS depletion by Tiron cannot prevent microvilli degradation, but is able to inhibit plasma membrane damage upon A1 treatment. (c) Actin inhibitors treatment did not prevent A1 cytotoxicity on $\mathrm{hESC}$ in addition to Tiron treatment. (d) Actin inhibitors treatment did not prevent A1-induced ROS production regardless of Tiron treatment. Before A1 treatment, cells were treated with phosphate-buffered saline (PBS) or the ROS scavenger (Tiron $50 \mathrm{mM}$ ) for $1 \mathrm{~h}$, followed by incubation with the actin inhibitors: latrunculin A (4 $\mu \mathrm{g})$, cytochalasin B $(4 \mu \mathrm{g})$ and cytochalasin D $(4 \mu \mathrm{g})$ for 5 min before A1 treatment. (e) Proposed mechanistic model of A1-induced hESC death 
ROS production occurs upstream of actin reorganization. It was reported previously that NADPH oxidase-derived ROS can mediate actin reorganization through the regulation of certain Rho GTPase family members. ${ }^{29}$ Therefore, it is likely that the massive actin reorganization was directly caused by excess ROS production. Consequently, the mechanical force generated by the massive actin reorganization could lead to plasma membrane damage.

\section{Discussion}

Cytotoxic A1 was shortlisted from a panel of mAbs generated against $h E S C$ with the aim of preventing teratoma formation in hESC-based therapy. A1 is cytotoxic to hESC but not differentiated cells and it recognizes an O-linked glycan epitope expressed on multiple antigens on hESC.

Mechanistically, A1 kills hESC via oncosis, not apoptosis, featuring rapid killing rate, cell swelling and plasma membrane damage. A1-induced oncosis was initiated by bivalent A1 binding to surface microvilli and dimerizing antigen receptors. Upon activation of death signaling, A1-treated hESC undergo microvilli degradation and homotypic adhesion at the early stage. This was followed by excess ROS production, which is upstream of massive actin reorganization, mitochondrial impairment and severe plasma membrane damage. Elevated ROS production is essential for A1-induced hESC death, which is produced by NADPH oxidase, most likely Nox2 isoform. Taken together, we proposed a mechanistic model of A1-induced hESC death (Figure 8e). To our knowledge, this is the first mechanistic model for antibody-induced oncosis on hESC.

So far, most of the findings on A1-induced hESC death were consistent with GA101-induced lymphoma cell death, ${ }^{18,24,23}$ suggesting that different $m A$ bs can induce oncosis via similar signaling pathways. Intriguingly, GA101-induced actin reorganization occurs upstream of homotypic adhesion and ROS production. This divergence might be explained by the difference in microvilli characteristics between hESC and lymphoma cells. Lymphoma cells have a median surface density and microvillar length ranging from 2 to 4 microvilli per $\mu \mathrm{m}^{2}$ and $0.3-0.4 \mu \mathrm{m} .{ }^{30}$ However, based on the SEM images (Figure 4a), hESC microvilli are generally two times denser and longer. Microvilli have been reported to initiate signaling pathways through surface receptors. ${ }^{31,32}$ In our study, predominant A1 binding on microvilli has suggested that A1induced hESC death might be initiated through microvilli. Similarly, GA101 might induce death signaling through microvilli on lymphoma cells.

In fact, latrunculin treatment on lymphoma cells was found to result in shortening or disappearance of microvilli. ${ }^{30}$ Therefore, in GA101 studies, prevention of homotypic adhesion, ROS production and eventual lymphoma cell death by latrunculin B treatment could be due to the lack of microvilli to initiate death signaling. However, with denser and longer microvilli on hESC, actin inhibitors within a non-toxic range might not be able to affect signaling through microvilli shortening.

This hypothesis about signaling through microvilli might also explain the difference in the time required for $\mathrm{mAb}$-induced cell death by GA101 (4 h) and A1 (5 min) since theoretically denser and longer microvilli would provide more receptors and therefore faster signal amplification. Moreover, microvilli are abundant with cellular adhesion proteins. Microvilli structure was also shown to be associated with homotypic adhesion of lymphoma cells. ${ }^{33}$ Hence, $\mathrm{A} 1$-induced restructuring of microvilli is likely the cause for the subsequent homotypic adhesion.

The molecular basis underlying the activation of Nox2NADPH oxidase upon $\mathrm{A} 1$ binding remains to be elucidated. It was known that different activation pathways of NADPH oxidase eventually converge at a common passage with the phosphorylation of p47phox and the activation of Rac. ${ }^{34}$ Therefore, the responsible kinase and guanine nucleotide exchange factors warrants further investigation. This common passage might also explain why different antibodies targeting different cell types can induce ROS production and eventually lead to oncosis. In addition, based on three well-studied pathways of Nox2-NADPH oxidase activation, G-protein coupled receptor (GPCR) or receptor tyrosine kinase (RTK) are involved in signal transduction across the plasma membrane. ${ }^{34}$ Activation of GPCR or RTK requires ligand binding mediated conformational change or dimerization, suggesting the importance of A1 bivalency. Previously, bivalent antibody-induced activation of GPCR $^{35}$ and RTK have been reported. ${ }^{36}$ Moreover, based on our previous study of engineered mAb84 fragments, antibody cytotoxicity was not only dependent on bivalency but also on the conformational interaction between mAb and antigen. Two bivalent scFv constructs with different linker length and flexibility were tested but only one was able to recapitulate the cytotoxicity of mAb84 on hESC. ${ }^{37}$ The specificity of antibody-antigen interaction was also observed for different anti-CD20 antibodies despite having overlapping epitopes, thus resulting in the classification of type I and II anti-CD20 mAbs based on the mode of lymphoma cell death. ${ }^{38}$ The same study suggested that the specificity is caused by differences in the orientation of antibody-antigen binding and the resultant membrane compartmentalization of antigen dimers. Therefore, it is imperative to identify the complete epitope that $A 1$ recognizes and delineate whether one or multiple receptors are required to trigger the signaling cascade.

NADPH oxidases are membrane bound and found at two major subcellular locations: plasma membrane ${ }^{39,40}$ and lysosomal membrane. ${ }^{41-43}$ As targeting to specific subcellular site is required for localized ROS production, knowing the site of A1-induced ROS production could provide insight to understanding the activation pathway of $\mathrm{NADPH}$ oxidase. Plasma membrane-bound NADPH oxidases orientate in the way that the electron transfers from cytoplasmic NADPH to oxygen on the extracellular side of the plasma membrane. Superoxide generated in the extracellular domain would be diluted by the larger volume and would have to diffuse back into the intracellular space. Hence, it is unlikely that this route could induce a rapid and drastic killing. Moreover, superoxide released into the extracellular domain would result in a homogeneously oxidized environment for all cells in the assay, which contradicts the direct correlation between hESC death and ROS production. Hence, superoxide is more likely produced in an intracellular compartment. As lysosomal membrane is formed from endocytosis of the plasma membrane, NADPH oxidase orientates in the opposite way 
so that superoxide is produced into the lumen of lysosome. Lysosomal-produced ROS has been implicated in different cell death pathways, including apoptosis, oncosis and autophagic cell death. ${ }^{44,45}$ Studies have also reported the link between superoxide production and lysosome membrane permeabilization (LMP), although it appears that superoxide can be both a cause and a consequence of LMP.44,46 Therefore, whether there is LMP in A1-treated hESC and how is LMP linked to A1induced ROS production warrants further investigation.

In conclusion, this is the first mechanistic model for mAbinduced hESC oncosis, which reveals a previously unrecognized role for NADPH oxidase-derived ROS in mediating oncotic hESC death. Combination of $A 1$ with ROS-generating reagents could be explored to provide synergistic cell killing in hESC. Further investigation of A1-induced hESC death pathway will be helpful in optimizing the efficacy of A1-based elimination of undifferentiated hESC. Collectively with previously reported role of NADPH oxidase-derived ROS in GA101-induced lymphoma death, it might support that NADPH oxidase-derived ROS has a central role in mediating $\mathrm{mAb}$-induced oncosis in various cell types.

\begin{abstract}
Materials and Methods
Cell culture. Human embryonic stem cell line, HES-3, was obtained from ES Cell International (ESI, Singapore; http://escellinternational). The cells were cultured at $37{ }^{\circ} \mathrm{C}$ with $5 \% \mathrm{CO}_{2}$ on Matrigel-coated culture dishes supplemented daily with conditioned media (CM) from immortalized mouse feeders, $\Delta \mathrm{E}-\mathrm{MEF}{ }^{47}$ The media used for culturing hESC was knockout (KO) media, which contained $85 \% \mathrm{KO}$ DMEM (Dulbecco's modified Eagle's medium) supplemented with 15\% KO serum replacer, $1 \mathrm{mM}$ L-glutamine, $25 \mathrm{U} / \mathrm{ml}$ penicillin, $25 \mathrm{U} / \mathrm{ml}$ streptomycin, $1 \%$ nonessential amino acids (NEAA), $0.1 \mathrm{mM}$ 2-mercaptoethanol and $5 \mathrm{ng} / \mathrm{ml}$ of recombinant human fibroblast growth factor-2 (FGF-2) (Invitrogen, Carlsbad, CA USA; http://www.invitrogen.com). Human iPSCs, ESIMR90, reprogrammed from fetal lung fibroblast, ${ }^{48}$ were cultured as described above for hESC, with the exception that $100 \mathrm{ng} / \mathrm{ml}$ instead of $5 \mathrm{ng} / \mathrm{ml}$ of FGF-2 was supplemented to the CM.

To induce hESC differentiation in vitro, $\mathrm{hESC}$ were mechanically cut and harvested as clumps and cultured as EBs for 7 days in EB media (80\% KO-DMEM, $20 \%$ fetal bovine serum, $25 \mathrm{U} / \mathrm{ml}$ penicillin, $25 \mu \mathrm{g} / \mathrm{ml}$ streptomycin, $2 \mathrm{mM} \mathrm{L-glutamine,}$ $0.1 \mathrm{mM}$ NEAA and $0.1 \mathrm{mM}$ 2-mercaptoethanol) on non-adherent suspension culture dishes (Corning, NY, USA). Subsequently, EBs were dissociated with trypsin, plated on gelatinized culture dishes ${ }^{49}$ and passaged every 7 days at an $1: 4$ ratio. EB media was replaced once every 2 days. See Supplementary Experimental Procedures for full details.
\end{abstract}

Fragmentation of A1. Papain digestion of lgG produces Fab fragments in the presence of reducing agent. In the experiments, $50 \mu \mathrm{l}$ of $2 \mathrm{mg} / \mathrm{ml}$ purified IgG A1 in PBS was incubated with $100 \mu \mathrm{l}$ of $0.1 \mathrm{mg} / \mathrm{ml}$ papain (Sigma Aldrich, St. Louis, MO, USA) in freshly made digestion buffer (PBS containing $0.02 \mathrm{M}$ EDTA, $0.02 \mathrm{M}$ cysteine) for $1 \mathrm{~h}$ in a $37^{\circ} \mathrm{C}$ water bath. After which, the mixture was removed from the water bath and the digestion reaction was terminated by adding $20 \mu \mathrm{l}$ of $0.3 \mathrm{M}$ iodoacetamide in PBS and vortexing. The reaction mixture was then dialyzed using VIVASPIN 500 (Sartorius Stedim Biotech, Goettingen, Germany) with membrane pore size of $30 \mathrm{kDa}$. $F(a b)_{2}$ fragments of $A 1$ were produced using IdeS Protease (Promega, Madison, WI, USA). Purified A1 was mixed with IdeS Protease in the ratio of $1 \mu \mathrm{g}$ of antibody to $1 \mathrm{U}$ of IdeS Protease in the digestion buffer. Mixture was incubated at $37^{\circ} \mathrm{C}$ for $45 \mathrm{~min}$. The efficiency of enzyme digestion as well as the size of different fragments was verified on SDS-PAGE with Coomassie blue staining and/ or western blotting.

Immunoprecipitation. Protein lysates in $2 \%$ Triton-100/PBS were used for immunoprecipitation (IP) on the automated MEA system (PhyNexus Inc., San Jose, CA, USA). Briefly, A1 $(\sim 100 \mu \mathrm{g})$ was directly captured onto Protein G PhyTip columns $(5 \mu \mathrm{l}$ resin bed; PhyNexus Inc.). After washing away the unbound proteins with PBS, clarified cell lysate was passed through the column functionalized with A1. The column was further washed with PBS, after which bound proteins were eluted at low pH with the Elution buffer $\left(200 \mathrm{mM} \mathrm{NaH}_{2} \mathrm{PO}_{4} / 140 \mathrm{mM} \mathrm{NaCl}, \mathrm{pH} 2.5\right)$ and neutralized immediately with $1 \mathrm{M}$ Tris- $\mathrm{Cl}(\mathrm{pH} 9.0)$.

SDS-PAGE and western blot. Protein lysates (20 $\mu \mathrm{g}$ per well) were separated by SDS-PAGE (NuPAGE 4-12\% gradient gel; Invitrogen, Carlsbad, CA, USA) under reducing ( $15 \% \beta$-mercaptoethanol) or non-reducing conditions followed by western blotting. For western blotting, resolved proteins were transferred onto PVDF membrane (Millipore, Billerica, MA, USA) at $100 \mathrm{~V}$ for $90 \mathrm{~min}$ and immunoblotted with appropriately diluted antibodies in PBS containing $2.5 \%$ BSA and $0.05 \%$ Tween-20, followed by HRP-conjugated antibodies (1:10 000 dilution; Dako, Santa Clara, CA, USA) or near-infrared fluorescence antibodies (1:1000 dilution; LI-COR, Lincoln, NE, USA). Protein bands were detected with either chemiluminescence from chemical reaction with ECL Prime (GE Healthcare Life Science, Chicago, IL, USA) using Medical X-ray processor 2000 (Kodak, Rochester, NY, USA) or Odyssey fluorescence scanner (LI-COR), respectively. See Supplementary Experimental Procedures for full details.

Light and fluorescence microscopy imaging. Detection of ROS production and homotypic adhesion upon A1 treatment were performed using an Olympus IX71 inverted microscope (Olympus, Shinjuku, Tokyo, Japan) in 96-well plates (Greiner CellStar, Kremsmunster, Austria). Fluorescence imaging of F-actin and G-actin upon A1 treatment was acquired using Spinning Disk Confocal (Perkin-Elmer, Waltham, MA, USA) in $\mu$-Dish ${ }^{35} \mathrm{~mm}$ high (ibidi). F-actin was labeled with Alexa Fluor 488 Phalloidin (Life Technologies, Waltham, MA, USA), nucleus was stained by Hoechst blue (Life Technologies) and G-actin was labeled with deoxyribonuclease I, Alexa Fluor 594 Conjugate (Life Technologies).

Scanning electron microscopy. After A1 treatment, unbound $A 1$ were washed away and cells were primarily fixed by $4 \%$ paraformaldehyde and $2.5 \%$ glutaraldehyde in PBS $(\mathrm{pH} 7.3)$ for $15 \mathrm{~min}$ at room temperature. Fixed cells were then washed and resuspended with deionized water and loaded onto poly-L-lysinecoated coverslips (20 $\mathrm{mm}$ in diameter). Cells were allowed to settle down for 10-15 min. Subsequently, cells were fixed with $4 \%$ paraformaldehyde and $2.5 \%$ glutaraldehyde ( $\mathrm{pH} 7.3$, in PBS) for $3-5 \mathrm{~h}$. Fixed cells were dehydrated in a graded series of ethanol from $30 \%$ to $100 \%$. Subsequently, dehydrated cells were dried by Critical Point Dryer (Tousimis Autosamdri-815, Rockville, MD, USA) and surface coated with Platinum (40 s, 40 mA; JEOL JFC-1600 Fine Coater, Akishima, Tokyo, Japan). Coated samples were examined with a SEM (Helios NanoLab DualBeam, FEI, Hillsboro, OR, USA) and cell morphology was observed under secondary electron mode. For ROS depletion experiment, cells were firstly preincubated with ROS scavenger Tiron before A1 treatment. For immuno-SEM, cells were primarily fixed with only $4 \%$ paraformaldehyde for $15 \mathrm{~min}$. After primary fixation, unreacted aldehyde was blocked away with $100 \mathrm{mM}$ glycine. After washing three times with $1 \%$ BSA/PBS, cells were incubated with $100 \mu$ l gold-conjugated secondary antibody $(25 \mathrm{~nm}$, goat anti-human, IgG, EMS, Hatfield, PA, USA) for $1 \mathrm{~h}$. Cells were then washed and fixed with $4 \%$ paraformaldehyde and $2.5 \%$ glutaraldehyde in PBS (pH 7.3) for $2 \mathrm{~h}$ before loaded to poly-L-lysine-coated coverslips (10 $\mathrm{mm}$ in diameter). After dehydration and critical point drying as describe above, cells on coverslips were surface coated with carbon. Coated samples were examined with a SEM (JEOL JSM-6701 F, FEG, Akishima, Tokyo, Japan). Cell morphology was observed under secondary electron mode, gold particles were detected under backscatter mode and a combined mode is available to overlay cell morphology with gold particles.

Induction of apoptosis via UV irradiation. Confluent hESC cultures were exposed to $200 \mathrm{~mJ}$ of UV using a UV crosslinker (UV Stratalinker 1800; Stratagene, Santa Clara, CA, USA) to induce apoptosis. ${ }^{13}$ After UV exposure, the cells were maintained at $37^{\circ} \mathrm{C} / 5 \% \mathrm{CO}_{2}$ for $20 \mathrm{~h}$ before harvesting for apoptotic assays. ${ }^{13}$

Caspases assay. Guava Caspase-3/7-FAM Kit and Caspase-9-SR Kit (Millipore, Billerica, MA, USA) were used to measure caspase-3/7/9 activity. Membrane integrity and cell viability were simultaneously evaluated using 7-AAD. Cells (non-treated, UV-treated, A1-treated) were resuspended in $100 \mu \mathrm{l}$ PBS at a concentration of $2 \times 10^{6}$ cells per $\mathrm{ml}$ and incubated with $10 \mu \mathrm{l}$ of caspase reagent for $1 \mathrm{~h}$ at $37^{\circ} \mathrm{C}$ in a $\mathrm{CO}_{2}$ incubator. At the end of incubation, cells were washed two times with provided washing buffer, resuspended in $200 \mu$ l of 7-AAD working solution and incubated for $10 \mathrm{~min}$ at room temperature. After incubation, cells were washed two times and analyzed by flow cytometer (Guava EasyCyte; Millipore). 
TUNEL assay. TUNEL assay measures the extent of DNA fragmentation upon cell death. ${ }^{50}$ APO-DIRECT TUNEL Kit (Millipore) was used. Cells (non-treated, UV-treated, A1-treated) were fixed in 1\% paraformaldehyde in PBS (pH 7.4) at a concentration of $1-2 \times 10^{6}$ cells per $\mathrm{ml}$ and the suspension was placed on ice for 30-60 min. After cells were washed two times with PBS, they were resuspended in $70 \%$ ethanol at a concentration of $1-2 \times 10^{6}$ cells per $\mathrm{ml}$ and incubated for $1 \mathrm{~h}$ on ice. Subsequently, cells were washed three times and resuspended in $50 \mu \mathrm{l}$ of staining solution ( $10 \mu \mathrm{l}$ of TdT reaction buffer, $0.75 \mu \mathrm{l}$ of TdT enzyme, $8 \mu \mathrm{l}$ of fluorescein-dUTP and $32.25 \mu$ lof distilled $\mathrm{H}_{2} \mathrm{O}$ ). Cells were then incubated for $1 \mathrm{~h}$ at $37^{\circ} \mathrm{C}$ and resuspended every $15 \mathrm{~min}$. After incubation, cells were washed with rinsing buffer, resuspended in $0.5 \mathrm{ml}$ of the propidium iodide/RNaseA solution, incubated in the dark for $30 \mathrm{~min}$ at room temperature and analyzed by flow cytometer (FACScan; Becton Dickinson FACS Calibur, BD Biosciences, Franklin Lakes, NJ, USA).

Antibody and inhibitors treatment. For mAb treatment experiments, single-cell suspension were harvested and resuspended in 1\% BSA/PBS. Cells $\left(2 \times 10^{5}\right)$ were treated with $5 \mu \mathrm{g}$ of mAb, PBS as buffer control, for 45 min at $4{ }^{\circ} \mathrm{C}$ in the volume of $100 \mu \mathrm{l}$ and then proceed for flow cytometry analysis.

A1 cytotoxicity on hESC was not affected at room temperature or at $37^{\circ} \mathrm{C}$. This was consistent with previously reported mAb84. ${ }^{12}$

In sugar blocking assay, $5 \mu \mathrm{g}$ of $\mathrm{A} 1$ was preincubated with nine different sugars in the concentration of $2 \mathrm{mM}$ in PBS (H1, H2, LNFP1, type-1 A (bgA), type-1 B (bgB), LewisA, LewisB, LewisX and LewisY; Dextra Laboratories Ltd, Reading, RG6 6BZ, UK) separately before the addition to $h E S C$.

For other inhibitor assays, where appropriate, hESC were preincubated with the actin polymerization inhibitors: latrunculin A (4 $\mu \mathrm{g}$; Sigma-Aldrich), cytochalasin B ( $4 \mu \mathrm{g}$; Sigma-Aldrich) and cytochalasin D ( $4 \mu \mathrm{g}$; Sigma-Aldrich) for $5 \mathrm{~min}$ before mAb treatment; or the ROS scavengers: Tiron (50 mM; Sigma-Aldrich) or Tempol (120 mM; Sigma-Aldrich) for $1 \mathrm{~h}$ before mAb treatment; or the NADPH oxidase inhibitors: DPI (240 $\mu \mathrm{M}$; Sigma-Aldrich), or APO (Apocynin, $40 \mathrm{mM}$; Sigma-Aldrich), or MPA (3.12 mM; Sigma-Aldrich) for $1 \mathrm{~h}$ before $\mathrm{mAb}$ treatment. For in-culture glycan inhibitor treatment, tunicamycin ( $5 \mu \mathrm{g} / \mathrm{ml}$; Sigma-Aldrich) and benzyl 2-acetamido-2deoxy- $\alpha$-D-galactopyranoside (4 mM; Sigma-Aldrich) were spiked into hESC culture in 6-well flat-bottom plate (Falcon, Corning, NY, USA) 4 days after passaging. Besides Tiron and Tempol, all other inhibitors were reconstituted in DMSO. Therefore, for negative control, hESC were treated with the same volume of DMSO as the inhibitors treatment.

Detection of ROS. ROS production was detected with HE staining (SigmaAldrich). Cells after preincubation with ROS scavengers and A1 treatment were resuspended in $200 \mu \mathrm{l} 1 \% \mathrm{BSA} / \mathrm{PBS}$ with $25 \mu \mathrm{M} \mathrm{HE}$ and incubated for $30 \mathrm{~min}$ in the dark at room temperature. After incubation, the intensity of red fluorescence was directly analyzed on a Guava InCyte Flow Cytometer (Merck Millipore) with detection emission in the Red-HLog channel. Alternatively, $2 \times 10^{5} \mathrm{hESC}$ were preincubated with $30 \mu \mathrm{M}$ carboxy-H2DCFDA (Life Technologies) in $100 \mu \mathrm{l}$ of $1 \%$ BSA/PBS for $10 \mathrm{~min}$ at $37^{\circ} \mathrm{C}$, and then transferred into a flat-bottomed 96-well plate (BD Science). Cells were imaged before and after treatment with $5 \mu \mathrm{g}$ of A1.

Assessment of homotypic adhesion formation. Human ESCs $\left(2 \times 10^{5}\right)$ were preincubated with $50 \mathrm{mM}$ Tiron or PBS in $100 \mu \mathrm{l} 1 \%$ BSA/PBS in a flat-bottomed 96-well plastic plate (BD Biosciences, Franklin Lakes, NJ, USA) for $1 \mathrm{~h}$ at $4{ }^{\circ} \mathrm{C}$. After preincubation, cells were treated with $5 \mu \mathrm{g}$ of $\mathrm{A} 1$ or $\mathrm{F}(\mathrm{ab})_{2} \mathrm{~A} 1$ or Fab_A1 or PBS for $45 \mathrm{~min}$ at $4{ }^{\circ} \mathrm{C}$. Subsequently, cells were viewed using light microscopy.

Measurement of NADPH oxidase activity. Human ESCs with or without A1 treatment were washed and pelleted with $2 \times 10^{6}$ cells in a microcentrifuge tube for NADP/NADPH Assay (Abcam, Cambridge, UK). Total NADP/NADPH was extracted from each sample according to the manufacturer's protocol. NADPH was obtained by decomposing the NADP component with heat. To quantify the concentration of total NADP/NADPH or NADPH, a standard curve was generated with provided NADPH standards. The average absorbance of the duplicates for each standard or sample was determined and normalized against the mean absorbance value of the blank. Based on the trendline equation of the standard curve, the concentration of total NADP/NADPH or NADPH of the samples was obtained. The concentration of NADP was obtained by subtracting the concentration of NADPH from that of total NADP/NADPH.
Knockdown of Nox2/gp91 and Nox3 using siRNA. siRNA reagents targeting Nox2 (S3787, S3788) or Nox3 (S27017), or scramble control siRNA were purchased from Life Technologies and delivered into $1 \times 10^{6}-2 \times 10^{6} \mathrm{hESC}$ at a final concentration of $120 \mathrm{pmol}$ using Lipofectamine RNAimax (Life Technologies) according to the manufacturer's instructions. See Supplementary Experimental Procedures for full details.

Mitochondrial membrane permeability assay. The membranepermeable JC-1 dye (Molecular Probes, Eugene, OR, USA) was used to assess the mitochondrial membrane permeability. JC-1 dye accumulation in the mitochondria is potential-dependent. JC-1 monomers emit green fluorescence $(\sim 529 \mathrm{~nm})$, whereas JC-1 aggregates emit red fluorescence $(\sim 590 \mathrm{~nm})$. Mitochondrial depolarization is indicated by a decrease in the red/green fluorescence intensity ratio. After A1 treatment, cells with or without Nox2 knockdown were washed and incubated with $\mathrm{JC}-1$ dye $\left(1: 100\right.$ dilutions) at $37^{\circ} \mathrm{C}$ for $30 \mathrm{~min}$. After incubation, red and green fluorescence changes were monitored with flow cytometry analysis.

Statistics. Error bars represent the standard error of mean (S.E.M.) of at least three independent experiments, unless otherwise stated. Significance in difference between the experimental groups was calculated using unpaired, two-tailed $t$-test. The degree of statistical significance was represented by the number of asterisks displayed on the figures determined by $P$-values as follows: ${ }^{*}<0.05,{ }^{*}<0.01$ and ${ }^{* * *}<0.001$.

\section{Conflict of Interest}

The authors declare no conflict of interest.

Acknowledgements. This work was supported by Bioprocessing Technology Institute (BTI), Agency for Science, Technology and Research (A*STAR), Singapore; Center for Biological Science (CBIS), National University of Singapore, Singapore; and NUS Graduate School for Integrative Science and Engineering, National University of Singapore, Singapore. We thank Dr. Vanessa Ding, Dr. Leung Hau Wan, Jayanthi Padmanabhan, Angela Chin, Lim Yu Ming, Dr. Alan Chan and Gerine Tong for their assistance or advice with tissue culture; Zheng Lu and Ally Lau for their assistance or advice with mass spectrometry; Charlene Yong and Chen Hui Shan for their assistance with RNA work; Loy Gek Luan and Chong Ping Lee for their assistance or advice with electron microscopy; Tong Yan for her assistance with confocal microscopy; Dr Julien Maury, Matthew Choo and Simeon Cua for their fruitful discussion and helpful suggestions.

1. Liu B, Fang M, Schmidt M, Lu Y, Mendelsohn J, Fan Z. Induction of apoptosis and activation of the caspase cascade by anti-EGF receptor monoclonal antibodies in DiFi human colon cancer cells do not involve the c-jun N-terminal kinase activity. Br J Cancer 2000; 82 . 1991-1999.

2. Tortora G, Caputo R, Pomatico G, Pepe S, Bianco AR, Agrawal S et al. Cooperative inhibitory effect of novel mixed backbone oligonucleotide targeting protein kinase $\mathrm{A}$ in combination with docetaxel and anti-epidermal growth factor-receptor antibody on human breast cancer cell growth. Clin Cancer Res 1999; 5: 875-881.

3. Huang SM, Harari PM. Modulation of radiation response after epidermal growth factor receptor blockade in squamous cell carcinomas: inhibition of damage repair, cell cycle kinetics, and tumor angiogenesis. Clin Cancer Res 2000; 6: 2166-2174.

4. Bazil V, Brandt J, Tsukamoto A, Hoffman R. Apoptosis of human hematopoietic progenitor cells induced by crosslinking of surface CD43. Blood 1995;86: 502-511.

5. Pedersen IM, Buhl AM, Klausen P, Geisler $\mathrm{CH}$, Jurlander J. The chimeric anti-CD20 antibody rituximab induces apoptosis in B-cell chronic lymphocytic leukemia cells through a p38 mitogen activated protein-kinase-dependent mechanism. Blood 2002; 99: 1314-1319.

6. Ludwig DL, Pereira DS, Zhu Z, Hicklin DJ, Bohlen P. Monoclonal antibody therapeutics and apoptosis. Oncogene 2003; 22: 9097-9106.

7. Trauth $\mathrm{BC}$, Klas $\mathrm{C}$, Peters AM, Matzku S, Möller $\mathrm{P}$, Falk $\mathrm{W}$ et al. Monoclonal antibodymediated tumor regression by induction of apoptosis. Science 1989; 245: 301-305.

8. Ichikawa K, Liu W, Zhao L, Wang Z, Liu D, Ohtsuka T et al. Tumoricidal activity of a novel anti-human DR5 monoclonal antibody without hepatocyte cytotoxicity. Nat Med 2001; 7: 954-960.

9. Trump BF, Berezesky IK, Chang SH, Phelps PC. The pathways of cell death: oncosis, apoptosis, and necrosis. Toxicol Pathol 1997; 25: 82-88.

10. Majno G, Joris I. Apoptosis, oncosis, and necrosis. An overview of cell death. Am J Pathol 1995; 146: 3-15. 
11. Weerasinghe P, Buja LM. Oncosis: an important non-apoptotic mode of cell death. Exp Mol Pathol 2012; 93: 302-308.

12. Choo AB, Tan HL, Ang SN, Fong WJ, Chin A, Lo J et al. Selection against undifferentiated human embryonic stem cells by a cytotoxic antibody recognizing podocalyxin-like protein-1. Stem Cells 2008; 26: 1454-1463.

13. Tan HL, Fong WJ, Lee EH, Yap M, Choo A. mAb 84, a cytotoxic antibody that kills undifferentiated human embryonic stem cells via oncosis. Stem Cells 2009; 27: 1792-1801.

14. Klement E, Lipinszki Z, Kupihár Z, Udvardy A, Medzihradszky KF. Enrichment of O-GlcNAc modified proteins by the periodate oxidation - hydrazide resin capture approach. $J$ Proteome Res 2010; 9: 2200-2206.

15. De Freitas JCM Jr, Silva Bdu R, de Souza WF, de Araújo WM, Abdelhay ES, Morgado-Díaz $\mathrm{JA}$. Inhibition of N-linked glycosylation by tunicamycin induces E-cadherin-mediated cell-cel adhesion and inhibits cell proliferation in undifferentiated human colon cancer cells. Cance Chemother Pharmacol 2011; 68: 227-238.

16. Rodgers AK, Nair A, Binkley PA, Tekmal R, Schenken RS. Inhibition of CD44 N- and O-linked glycosylation decreases endometrial cell lines attachment to peritoneal mesothelial cells. Fertil Steril 2011; 95: 823-825.

17. Elmore S. Apoptosis: a review of programmed cell death. Toxicol Pathol 2007; 35: 495-516

18. Ivanov A, Beers SA, Walshe CA, Honeychurch J, Alduaij W, Cox KL et al. Monoclonal antibodies directed to CD20 and HLA-DR can elicit homotypic adhesion followed by lysosome-mediated cell death in human lymphoma and leukemia cells. J Clin Invest 2009; 119: 2143-2159.

19. Roque-Navarro L, Chakrabandhu K, de León J, Rodríguez S, Toledo C, Carr A et al. Anti-ganglioside antibody-induced tumor cell death by loss of membrane integrity. Mol Cancer Ther 2008; 7: 2033-2041.

20. Mone AP, Huang P, Pelicano H, Cheney CM, Green JM, Tso JY et al. Hu1D10 induces apoptosis concurrent with activation of the AKT survival pathway in human chronic lymphocytic leukemia cells. Blood 2004; 103: 1846-1854.

21. Bras M, Yuste VJ, Roué G, Barbier S, Sancho P, Virely $C$ et al. Drp1 mediates caspaseindependent type III cell death in normal and leukemic cells. Mol Cell Biol 2007; 27: 7073-7088.

22. Alinari L, Yu B, Christian BA, Yan F, Shin J, Lapalombella R et al. Combination anti-CD74 (milatuzumab) and anti-CD20 (rituximab) monoclonal antibody therapy has in vitro and in vivo activity in mantle cell lymphoma. Blood 2011; 117: 4530-4541.

23. Honeychurch J, Alduaij W, Azizyan M, Cheadle EJ, Pelicano H, Ivanov A et al. Antibodyinduced nonapoptotic cell death in human lymphoma and leukemia cells is mediated through a novel reactive oxygen species-dependent pathway. Blood 2012; 119: 3523-3533.

24. Alduaij W, Ivanov A, Honeychurch J, Cheadle EJ, Potluri S, Lim SH et al. Novel type II antiCD20 monoclonal antibody (GA101) evokes homotypic adhesion and actin-dependent, lysosome-mediated cell death in B-cell malignancies. Blood 2011; 117: 4519-4529.

25. Yamada J, Yoshimura S, Yamakawa H, Sawada M, Nakagawa M, Hara S et al. Cell permeable ROS scavengers, Tiron and Tempol, rescue PC12 cell death caused by pyrogallo or hypoxia/reoxygenation. Neurosci Res 2003; 45: 1-8.

26. Taiwo FA. Mechanism of tiron as scavenger of superoxide ions and free electrons. $J$ Spectrosc 2008; 22: 491-498.

27. Novo E, Parola M. Redox mechanisms in hepatic chronic wound healing and fibrogenesis. Fibrogenesis Tissue Rep 2008; $1: 5$.

28. Düssmann H, Kögel D, Rehm M, Prehn JHM. Mitochondrial membrane permeabilization and superoxide production during apoptosis a single-cell analysis. J Biol Chem 2003; 278 : 12645-12649.

29. Stanley A, Thompson K, Hynes A, Brakebusch C, Quondamatteo F. NADPH oxidase complex-derived reactive oxygen species, the actin cytoskeleton, and Rho GTPases in cell migration. Antioxid Redox Signal 2014; 20: 2026-2042.

30. Majstoravich S, Zhang J, Nicholson-Dykstra S, Linder S, Friedrich W, Siminovitch KA et al. Lymphocyte microvilli are dynamic, actin-dependent structures that do not require Wiskott-Aldrich syndrome protein (WASp) for their morphology. Blood 2004; 104 1396-1403.

31. Juang SH, Carvajal ME, Whitney M, Liu Y, Carraway CA. Tyrosine phosphorylation at the membrane-microfilament interface: a p185neu-associated signal transduction particle containing Src, Abl and phosphorylated p58, a membrane- and microfilament-associated retroviral gag-like protein. Oncogene 1996; 12: 1033-1042.

32. Menco BPM. The fine-structural distribution of G-protein receptor kinase 3, beta-arrestin-2, $\mathrm{Ca}^{2+} /$ calmodulin-dependent protein kinase II and phosphodiesterase PDE1C2, and a $\mathrm{Cl}$ (-)-cotransporter in rodent olfactory epithelia. J Neurocytol 2005; 34: 11-36.

33. Greicius G, Westerberg L, Davey EJ, Buentke E, Scheynius A, Thyberg J et al. Microvilli structures on B lymphocytes: inducible functional domains? Int Immunol 2004; 16 : 353-364.

34. Brandes RP, Kreuzer J. Vascular NADPH oxidases: molecular mechanisms of activation. Cardiovasc Res 2005; 65: 16-27.

35. Mijares A, Lebesgue D, Wallukat G, Hoebeke J. From agonist to antagonist: Fab fragments of an agonist-like monoclonal anti-beta(2)-adrenoceptor antibody behave as antagonists. Mol Pharmacol 2000; 58: 373-379.

36. Spaargaren M, Defize LH, Boonstra J, de Laat SW. Antibody-induced dimerization activates the epidermal growth factor receptor tyrosine kinase. J Biol Chem 1991; 266: 1733-1739.

37. Lim DYX, Ng YH, Lee J, Mueller M, Choo AB, Wong VV. Cytotoxic antibody fragments for eliminating undifferentiated human embryonic stem cells. J Biotechnol 2011; 153: 77-85.

38. Niederfellner G, Lammens A, Mundigl O, Georges GJ, Schaefer W, Schwaiger M et al. Epitope characterization and crystal structure of GA101 provide insights into the molecular basis for type I/II distinction of CD20 antibodies. Blood 2011; 118: 358-367.

39. Smith KR, Klei LR, Barchowsky A. Arsenite stimulates plasma membrane NADPH oxidase in vascular endothelial cells. Am J Physiol Lung Cell Mol Physiol 2001; 280: L442-L449.

40. Fisher AB. Redox signaling across cell membranes. Antioxid Redox Signal 2009; 11 : 1349-1356.

41. Bao J-X, Chang H, Lv YG, Yu JW, Bai YG, Liu H et al. Lysosome-membrane fusion mediated superoxide production in hyperglycaemia-induced endothelial dysfunction. PLoS One 2012; 7: $\mathrm{e} 30387$.

42. Bao J-X, Jin S, Zhang F, Wang ZC, Li N, Li PL. Activation of membrane NADPH oxidase associated with lysosome-targeted acid sphingomyelinase in coronary endothelial cells. Antioxid Redox Signal 2010; 12: 703-712.

43. Nohl H, Gille L. Lysosomal ROS formation. Redox Rep 2005; 10: 199-205.

44. Jäättelä M. Multiple cell death pathways as regulators of tumour initiation and progression. Oncogene 2004; 23: 2746-2756.

45. Kubota C, Torii S, Hou N, Saito N, Yoshimoto Y, Imai H et al. Constitutive reactive oxygen species generation from autophagosome/lysosome in neuronal oxidative toxicity. J Biol Chem 2010; 285: 667-674.

46. Boya P, Kroemer G. Lysosomal membrane permeabilization in cell death. Oncogene 2008; 27: 6434-6451.

47. Choo A, Padmanabhan J, Chin A, Fong WJ, Oh SKW. Immortalized feeders for the scale-up of human embryonic stem cells in feeder and feeder-free conditions. J Biotechnol 2006; 122: 130-141.

48. Zhang J, Wilson GF, Soerens AG, Koonce $\mathrm{CH}$, Yu J, Palecek SP et al. Functional cardiomyocytes derived from human induced pluripotent stem cells. Circ Res 2009; 104: e30-e41.

49. Heins N, Lindahl A, Karlsson U, Rehnström M, Caisander G, Emanuelsson K et al. Clonal derivation and characterization of human embryonic stem cell lines. J Biotechnol 2006; 122: 511-520.

50. Sen S, Jumaa H, Webster NJG. Splicing factor SRSF3 is crucial for hepatocyte differentiation and metabolic function. Nat Commun 2013; 4: 1336.

51. O'Donnell BV, Tew DG, Jones OT, England PJ. Studies on the inhibitory mechanism of iodonium compounds with special reference to neutrophil NADPH oxidase. Biochem J 1993; 290: $41-49$.

52. Krötz F, Keller M, Derflinger S, Schmid H, Gloe T, Bassermann F et al. Mycophenolate acid inhibits endothelial $\mathrm{NAD}(\mathrm{P}) \mathrm{H}$ oxidase activity and superoxide formation by a Rac1dependent mechanism. Hypertension 2007; 49: 201-208.

53. Stolk J, Hiltermann TJ, Dijkman JH, Verhoeven AJ. Characteristics of the inhibition of NADPH oxidase activation in neutrophils by apocynin, a methoxy-substituted catechol. Am J Respir Cell Mol Biol 1994; 11: 95-102.

\section{Supplementary Information accompanies this paper on Cell Death and Differentiation website (http://www.nature.com/cdd)}

